\title{
Half-Collision Approach to Cold Chemistry: Shape Resonances, Elastic Scattering, and Radiative Association in the $\mathrm{H}^{+}+\mathrm{H}$ and $\mathrm{D}^{+}+\mathrm{D}$ Collision Systems
}

\author{
Maximilian Beyer and Frédéric Merkt \\ Laboratorium für Physikalische Chemie, ETH Zürich, 8093 Zürich, Switzerland
}

(Received 12 April 2018; revised manuscript received 29 May 2018; published 27 September 2018)

\begin{abstract}
The $\mathrm{H}^{+}+\mathrm{H}$ and $\mathrm{D}^{+}+\mathrm{D}$ collisions represent the simplest examples of collisions involving identical fermions and bosons, respectively. We report a study of these collisions in the range of energies corresponding to $0-40 \mathrm{~K}$ at a resolution of $100 \mathrm{mK}$ based on a half-collision approach. Using the technique of pulsed-field-ionization zero-kinetic-energy photoelectron spectroscopy (PFI-ZEKE-PES), which relies on the pulsed field ionization of nonpenetrating Rydberg states of very high principal quantum number ( $n \geq 250$ ), we have measured the positions and widths of all low-lying shape resonances of this collision and compare them with the results of ab initio calculations that consider nonadiabatic, relativistic, and radiative corrections to the Born-Oppenheimer energies. Excellent agreement between calculated and measured widths and positions is found except for the $X^{+}\left(v^{+}=25, N^{+}=8\right)$ shape resonance, the width of which is calculated to be narrower than observed. Comparison with similar observations made for the $\mathrm{H}^{+}+\mathrm{H}$ collision [Beyer and Merkt Phys. Rev. Lett. 116, 093001 (2016)] indicates that the shape resonances measured by PFI-ZEKE-PES are broadened by the effects of the localization of the charge of the dissociating ion core arising from a nonadiabatic coupling between ion core and Rydberg electron. A simple model is presented that enables one to predict which shape resonances are likely to be affected and to correct for the resulting broadening. Two shape resonances of $\mathrm{D}_{2}{ }^{+}$, the $X^{+}\left(v^{+}=26, N^{+}=5\right)$ and the $A^{+}\left(v^{+}=0, N^{+}=5\right)$ resonances, have electric-dipole-allowed transitions to bound states of $\mathrm{D}_{2}{ }^{+}$and are shown to facilitate the radiative association at low temperature. The results are used to determine the elastic cross sections of the $\mathrm{H}^{+}+\mathrm{H}$ and $\mathrm{D}^{+}+\mathrm{D}$ collisions for partial waves up to $N^{+}=11$ and the radiative-association cross sections and rate constants in the range between $10 \mathrm{mK}$ and $100000 \mathrm{~K}$.
\end{abstract}

DOI: 10.1103/PhysRevX.8.031085

Subject Areas: Atomic and Molecular Physics

\section{INTRODUCTION}

Shape resonances play an important role in low-temperature scattering and their study represents an important aspect of the rapidly growing field of cold chemistry (see, e.g., Refs. [1-6]). The molecular hydrogen ion $\mathrm{H}_{2}{ }^{+}$and its deuterated and tritiated isotopomers represent the simplest molecular systems displaying shape resonances associated with the motion of the nuclei, i.e., quasibound rotational levels located energetically above the dissociation limit. Accurate calculations of these resonances are possible and offer the prospect of a detailed comparison with experimental results. Information on the shape resonances of the molecular hydrogen ion is also relevant for the determination of its partition function and of the $\mathrm{H}^{+}+\mathrm{H}$ elastic-scattering cross section, and for understanding the corresponding recombination reaction $\mathrm{H}^{+}+\mathrm{H} \rightarrow \mathrm{H}_{2}^{+}$,

Published by the American Physical Society under the terms of the Creative Commons Attribution 4.0 International license. Further distribution of this work must maintain attribution to the author(s) and the published article's title, journal citation, and DOI. one of the few chemical reactions of importance in the early Universe [7-13].

We report here on a half-collision approach to the full $\mathrm{H}^{+}+\mathrm{H}$ and $\mathrm{D}^{+}+\mathrm{D}$ quantum scattering and to the radiative-association reactions forming $\mathrm{H}_{2}{ }^{+}$and $\mathrm{D}_{2}{ }^{+}$, which has led to the determination of highly accurate scattering cross sections and reaction-rate coefficients over the temperature range from below $10 \mathrm{mK}$ to above $100000 \mathrm{~K}$. This approach relies on the following steps:

(1) Measurement of the positions and widths of the shape resonances of $\mathrm{H}_{2}{ }^{+}$and $\mathrm{D}_{2}{ }^{+}$,

(2) Full ab initio quantum-chemical calculations of the positions and widths of these resonances including all known effects,

(3) Comparison of the experimental and calculated results to validate the $a b$ initio calculations,

(4) Determination of the scattering phase shifts for individual partial waves of the $\mathrm{H}^{+}+\mathrm{H}$ and $\mathrm{D}^{+}+\mathrm{D}$ collisions,

(5) Determination of the absolute elastic-scattering cross sections and their energy dependence in the collision energy range from below $10 \mu \mathrm{eV}$ to above $10 \mathrm{eV}$, including all quantum effects, and 
(6) Determination of the absolute radiative-association cross section and the corresponding thermal rate coefficients over the complete temperature range indicated above, including all quantum effects.

This approach differs fundamentally from recent approaches to study ion-atom collisions at very low temperatures using trapped ions immersed in ultracold atomic vapors (see, e.g., Refs. [14-18]) but is applicable to a broader range of species. In particular, it enables the study of collisional and reactive processes involving the lightest atoms $\mathrm{H}^{+}$and $\mathrm{H}$, which are ideally suited for the investigation of quantum-mechanical effects.

Until recently, no experimental information was available on the shape resonances of the molecular hydrogen ion, primarily because of (i) the extreme difficulties associated with high-resolution, low-energy scattering experiments involving charged particles and open-shell atoms [19,20], and (ii) similar difficulties associated with high-resolution spectroscopic studies of long-range states of molecular ions [21-25], which, in the case of $\mathrm{H}_{2}{ }^{+}$and $\mathrm{D}_{2}{ }^{+}$, are exacerbated by the absence of a permanent electric dipole moment.

In the case of $\mathrm{H}_{2}{ }^{+}, 58$ shape resonances were predicted to exist by Moss [26], who calculated the positions of the 39 narrowest ones. In a theoretical study of the $\mathrm{H}^{+}+\mathrm{H}$ collision, Krstic et al. later reported 96 resonances in the elastic and charge-transfer cross sections, 34 of which correspond to shape resonances, 40 to top-of-the-barrier resonances, and 22 to above-barrier resonances [27]. Recently, we have observed the two lowest-lying shape resonances of $\mathrm{H}_{2}{ }^{+}[28,29]$, i.e., the $X^{+}\left(v^{+}=18, N^{+}=4\right)$ and the $X^{+}\left(v^{+}=17, N^{+}=7\right)$ shape resonances, using PFI-ZEKE-PES [30]. Here and below, we designate the molecular levels of $\mathrm{H}_{2}{ }^{+}$and $\mathrm{D}_{2}{ }^{+}$using the electronic state labels $\left(\mathrm{X}^{+}\right.$and $\left.\mathrm{A}^{+}\right)$followed by the vibrational $\left(v^{+}\right)$and rotational $\left(\mathrm{N}^{+}\right)$quantum numbers in parentheses. In collisional processes, $N^{+}$also represents the collision partialwave label. In PFI-ZEKE-PES, one monitors the field ionization of very high Rydberg states, located energetically just below the ionic quantum levels to which the Rydberg series converge, as a function of the frequency of a tunable laser, as explained in Ref. [31]. Subsequently, the positions and widths of all resonances of $\mathrm{H}_{2}{ }^{+}$were determined $a b$ initio in calculations that included nonadiabatic, relativistic and quantum-electrodynamic corrections $[28,29,32]$. Quantitative agreement was reached between calculated and experimental values of the positions of both resonances and of the width of the $(18,4)$ resonance, but the measured width of the $(17,7)$ resonance was found to be significantly larger $\left[\Gamma_{\mathrm{obs}}=0.56(8) \mathrm{cm}^{-1}\right]$ than predicted by the calculations $\left(\Gamma_{\text {calc }}=0.16 \mathrm{~cm}^{-1}\right)$. This disagreement represented an obstacle that prevented us from validating the $a b$ initio calculations and from carrying out the remaining steps (steps 4 to 6) of the halfcollision approach described above. To obtain more information on the causes of the disagreement just mentioned, we have now completed a study of the shape resonances of $\mathrm{D}_{2}{ }^{+}$.

Prior to this work, no information was available on the shape resonances of $\mathrm{D}_{2}{ }^{+}$. The $\mathrm{D}^{+}+\mathrm{D}$ collision was studied in the collision-energy range 5-100 eV [33]. Carrington and co-workers [21,22,24,25] reported the microwave electronic spectrum of the $A^{+}-X^{+}$band system of $\mathrm{D}_{2}{ }^{+}$and compared experimental and calculated frequencies of the transitions between the highest bound vibrational levels of the $X^{+}$state and the lowest levels of the $A^{+}$ state. Moss [34], and Wolniewicz and Orlikowski [35] reported the positions of all bound levels of $\mathrm{D}_{2}{ }^{+}$with rotational angular momentum $N^{+} \leq 5$ and 8 , respectively, including those of the seven bound states $\left[\left(v^{+}=0\right.\right.$, $\left.\left.N^{+}=0-4\right),\left(v^{+}=1, N^{+}=0,1\right)\right]$ of the $A^{+2} \Sigma_{u}^{+}$first excited state. Beyer and Merkt [36] measured the positions of 65 bound states of the $X^{+2} \Sigma_{g}^{+}$state with $v^{+} \geq 20$, and of five of the seven bound levels of the $A^{+}$state and found good agreement with the results of Moss [34], Wolniewicz and Orlikowski [35], and their own calculations.

In this article, we report the observation of the $X^{+}(25,8)$, $(25,9),(26,5)$, and $(24,11)$, and the $A^{+}(0,5)$ low-lying shape resonances of $\mathrm{D}_{2}{ }^{+}$and compare their positions and widths with those obtained in $a b$ initio calculations. We find that the positions and widths of the resonances are well reproduced by the calculations, with the exception of the width of the $X^{+}(25,8)$ shape resonance, which is observed to be significantly larger than predicted by the calculations. These new data enabled us to understand the cause of the discrepancies between observed and calculated widths in $\mathrm{H}_{2}{ }^{+}$and $\mathrm{D}_{2}{ }^{+}$and to validate the ab initio calculations. We could thus carry out the last steps of the half-collision approach outlined above and fully characterize the $\mathrm{H}^{+}+\mathrm{H}$ and $\mathrm{D}^{+}+\mathrm{D}$ collisions and the corresponding radiativeassociation reactions.

\section{EXPERIMENT}

The experiments were carried out using the equipment and methods described in Refs. [29,36]. A schematic representation of the experimental setup is depicted in Fig. 1. The region around the dissociative-ionization threshold of $\mathrm{D}_{2}$, which is located $146456.9775(7) \mathrm{cm}^{-1}$ above the $X^{1} \Sigma_{g}^{+}(v=0, N=0)$ ground state of $\mathrm{D}_{2}$ [37], was accessed using the resonant three-photon excitation sequence

$$
X(v=0) \stackrel{\mathrm{VUV}}{\rightarrow} B(25) \stackrel{\mathrm{VIS}}{\rightarrow} \bar{H}(12) \stackrel{\mathrm{UV}}{\rightarrow} \mathrm{D}^{+}+\mathrm{D}+e^{-}
$$

through excited rovibrational levels of the intermediate $B^{1} \Sigma_{u}^{+}$and $\bar{H}^{1} \Sigma_{g}^{+}$states using three pulsed nanosecond tunable lasers operating in the vacuum-ultraviolet (VUV), visible (VIS), and UV ranges of the electromagnetic spectrum. The laser radiations in these frequency ranges are generated using four commercial pulsed dye lasers pumped 


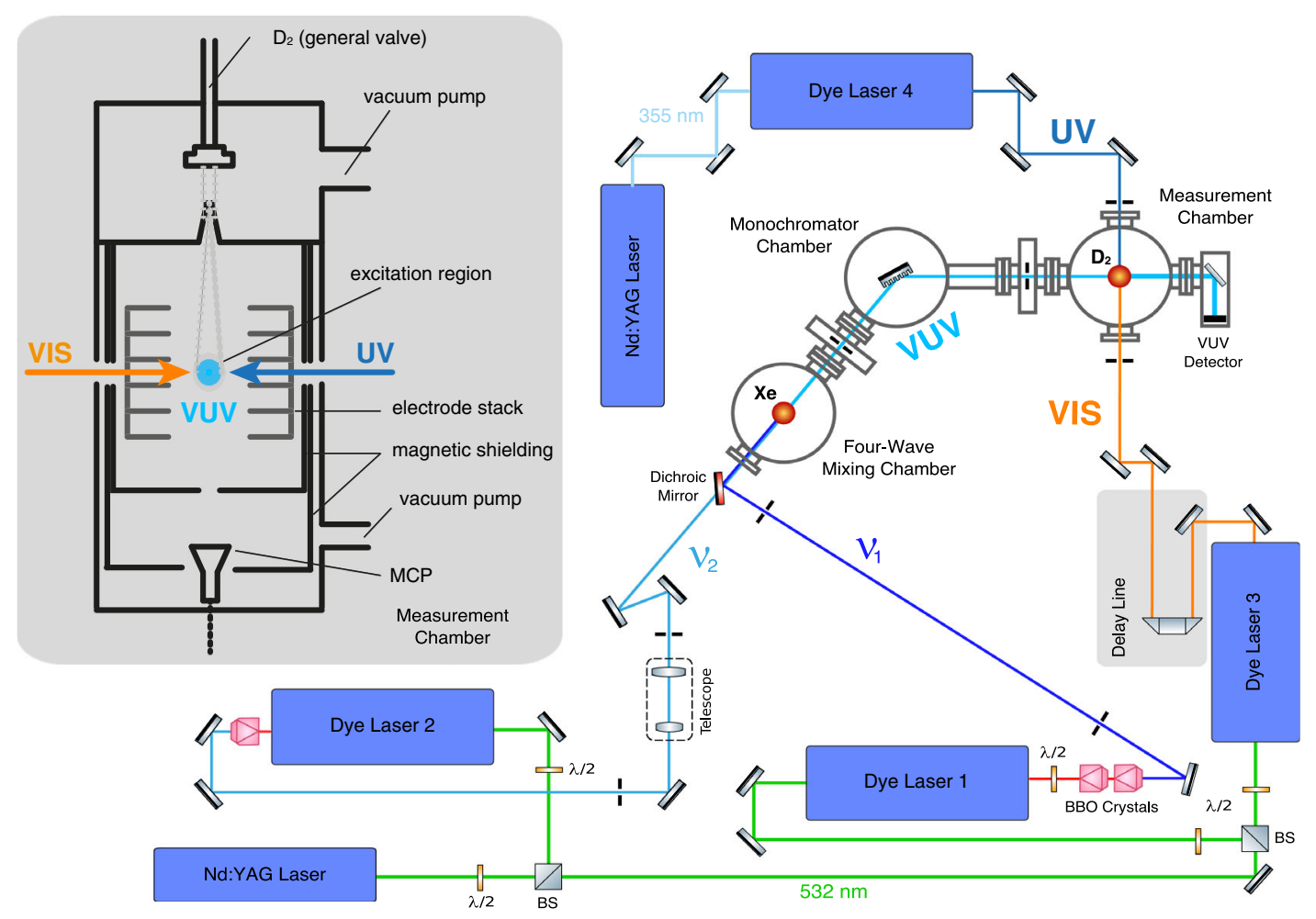

FIG. 1. Schematic representation of the experimental setup with the laser system, the vacuum chambers and the photoexcitation region.

by two different Nd:YAG lasers and several $\beta$-barium-borate (BBO) crystals for frequency up-conversion. The first two dye lasers are used to generate VUV radiation by two-photon resonant sum-frequency mixing $\left(\nu_{\mathrm{VUV}}=2 \nu_{1}+\nu_{2}\right)$ in a pulsed jet of the nonlinear gas Xe using the two-photon resonance at a wave number of $2 \nu_{1}=80118.984 \mathrm{~cm}^{-1}$. The sum-frequency mixing takes place in a four-wavemixing chamber that is separated from the measurement chamber by a monochromator chamber. The toroidal monochromator grating separates the VUV laser beam from the two input beams, recollimates it and directs it into the measurement region, where it crosses a pulsed (pulse length $200 \mu \mathrm{s})$ supersonic beam of the sample gas $\left(\mathrm{H}_{2}\right.$ or $\mathrm{D}_{2}$ ) at right angles. The other two lasers (labeled VIS and UV in Fig. 1) enter the measurement chamber through two opposite ports and also cross the supersonic beam of the sample gas at right angles. The VIS (laser 3 in Fig. 1) laser prepares a population in a selected rotational level of the $v=12$ level of the $\bar{H}$ state.

Spectra of the shape resonances of $\mathrm{D}_{2}{ }^{+}$were recorded from selected rotational levels of the $\bar{H}(v=12)$ intermediate state using a narrow-band UV laser, delayed by 20 ns with respect to the VUV and VIS lasers to avoid ionization from the short-lived $B(v=25)$ state, by monitoring the field-ionization yield of high-lying Rydberg states as a function of the wave number of the UV laser using the method of PFI-ZEKE PES.
To this end, electric-field pulse sequences were applied across a stack of six equally spaced cylindrical electrodes carefully designed to produce homogeneous fields and depicted in the gray-shaded inset of Fig. 1. The field ionization used to record the PFI-ZEKE PE spectra was induced by an electric-field pulse sequence consisting of ten successive steps $(1,-50 \mathrm{mV} / \mathrm{cm} ; 2,-70 \mathrm{mV} / \mathrm{cm} ; 3$, $-80 \mathrm{mV} / \mathrm{cm} ; 4,-90 \mathrm{mV} / \mathrm{cm} ; 5,-110 \mathrm{mV} / \mathrm{cm} ; 6$, $-130 \mathrm{mV} / \mathrm{cm} ; 7,-200 \mathrm{mV} / \mathrm{cm} ; 8,-270 \mathrm{mV} / \mathrm{cm} ; 9$, $-670 \mathrm{mV} / \mathrm{cm}$; and $10,-1.20 \mathrm{~V} / \mathrm{cm}$ ) generated with an arbitrary-function generator. The pulse sequence was typically applied one microsecond after the UV laser pulse to avoid the detection of prompt electrons. The electrons produced by the successive steps of the field-ionization sequence were extracted toward a microchannel-plate (MCP) detector and collected separately by setting temporal gates on the electron time-of-flight spectrum. The first step of the pulse sequence did not yield spectra of sufficient quality for subsequent analysis, so that nine PFI-ZEKE photoelectron spectra were obtained for each scan of the UV laser. The resolution of about $0.15 \mathrm{~cm}^{-1}$ of the spectra recorded with pulses 2-6 was primarily limited by the UV laser bandwidth. These spectra differ in the range of Rydberg states that are ionized by the successive pulses, which were designed so as to have maximum signal for Rydberg states with principal quantum numbers around $n_{\max }=330,310,295,280$, and 270, respectively, as 
explained in Ref. [38]. The resolution and the $n_{\max }$ value of the spectra recorded with the last steps of the sequence gradually decrease $\left(7,0.3 \mathrm{~cm}^{-1}, n_{\max }=260 ; 8,0.5 \mathrm{~cm}^{-1}\right.$, $n_{\max }=240 ; 9,1.0 \mathrm{~cm}^{-1}, \quad n_{\max }=220 ; 10,1.5 \mathrm{~cm}^{-1}$, $\left.n_{\max }=175\right)$.

The excitation region and the electron flight tube were surrounded by two concentric mumetal magnetic shields, and the stray electric fields in the laser-excitation region were reduced to below $5 \mathrm{mV} / \mathrm{cm}$.

\section{CALCULATIONS}

For the calculations of bound $\left(v^{+}, N^{+}\right)$and continuum $\left(k, N^{+}\right)$states of the electronic state $i$ the nuclear Schrödinger equation (in atomic units)

$$
\begin{aligned}
& {\left[-\frac{1}{2 \mu_{\mathrm{vib}}} \frac{d^{2}}{d R^{2}}+U+\frac{N^{+}\left(N^{+}+1\right)}{2 \mu_{\mathrm{rot}} R^{2}}-E_{v^{+} / k, N^{+}}^{i}\right]} \\
& \quad \times \phi_{v^{+} / k, N^{+}}^{i}(R)=0
\end{aligned}
$$

was solved, ensuring unit normalization for the bound state wave functions $\phi_{v^{+}, N^{+}}^{i}(R)$ and energy normalization for the continuum wave functions

$$
\begin{aligned}
& \lim _{R \rightarrow \infty} \phi_{k, N^{+}}^{i}(R) \\
& =\sqrt{\frac{2 \mu_{\mathrm{vib}}}{\pi k}} k R\left[j_{N^{+}}(k R) \cos \eta_{N^{+}}-n_{N^{+}}(k R) \sin \eta_{N^{+}}\right],
\end{aligned}
$$

where $j_{N^{+}}$and $n_{N^{+}}$are the Riccati-Bessel functions and $k=\sqrt{2 \mu_{\mathrm{vib}}\left[E_{k, N^{+}}^{i}-U(\infty)\right]}[29]$.

The first three terms in Eq. (2) represent the nuclearkinetic-energy operator, the effective potential for the nuclear motion $\left(U=U^{\mathrm{BO}}+\left\langle H_{1}^{\prime}\right\rangle+\left\langle H_{2}^{\prime}\right\rangle+\left\langle H_{\text {rel }}\right\rangle+\left\langle H_{\text {rad }}\right\rangle\right)$, and the centrifugal potential. The Born-Oppenheimer potential $U^{\mathrm{BO}}$ and the adiabatic correction terms $\left\langle H_{1}^{\prime}\right\rangle+$ $\left\langle H_{2}^{\prime}\right\rangle$ are calculated as explained in Sec. 4 of Ref. [29]. For the $X^{+}$state, nonadiabatic corrections are calculated approximately by using the $R$-dependent vibrational $\left(\mu_{\mathrm{vib}}\right)$ and rotational $\left(\mu_{\mathrm{rot}}\right)$ reduced masses reported by Jaquet [39], with which we obtain a closer agreement with the nonadiabatic energies determined for bound states by Moss [34] than with the reduced masses from Ref. [40] used in our previous study of $\mathrm{D}_{2}$ [36]. Dissociation energies including relativistic and radiative corrections are determined by adding the terms $\left\langle H_{\text {rel }}\right\rangle$ and $\left\langle H_{\text {rad }}\right\rangle$ to the adiabatic potential curves as described in Ref. [29], using the numerical values given in Ref. [32]. For the weakly bound $A^{+}$state, the relativistic and radiative corrections can be neglected in good approximation. The nonadiabatic corrections were taken into account approximatively by setting both $\mu_{\mathrm{vib}}$ and $\mu_{\mathrm{rot}}$ equal to $m_{d}\left(m_{d}+1\right) /\left(2 m_{d}+1\right)$, where $m_{d}$ is the deuteron mass.
To determine the positions and widths of the resonances, we repeated the procedure described in detail in Ref. [29] after replacing the proton mass by the deuteron mass. The procedure involves the numerical determination of the energy-dependent scattering phase shift $\eta_{N^{+}}(E)$ by matching the nuclear wave function to the expression in Eq. (3) in the asymptotic region, where all but the centrifugal term of the potential have become negligible. Resonance positions $E_{\text {res }}$ are extracted using the collision-time-delay approach developed by Smith [41], by locating the maxima of $\left[\left(d \eta_{N^{+}}\right) /(d E)\right]$. The corresponding widths are obtained as $\Gamma=2 /\left[\left(d \eta_{N^{+}}\right) /(d E)\right]_{E=E_{\mathrm{res}}}$.

Figure 2 presents the energy dependence of the phase shifts of the first 12 channels, corresponding to the $N^{+}=$ 0-11 partial waves. Each graph depicts the evolution of $\eta_{N^{+}}(E)$ modulo $\pi$ for both the $X^{+}$(blue traces) and $A^{+}$(red traces) states as a function of the dissociation energy, a negative energy thus implying a continuum state. The general energy dependence of the scattering phase shifts can be interpreted as follows: According to Levinson's theorem, the phase shift at zero energy is given by $\pi \mathcal{N}_{N^{+}}$, where $\mathcal{N}_{N^{+}}$is the total number of states with angular momentum $N^{+}$bound by the potential. The phase shift further increases up to the top of the centrifugal barrier or slightly above. In this range, it can be decomposed into $\eta_{N^{+}}=\eta_{N^{+}}^{\mathrm{HS}}+\eta_{N^{+}}^{\mathrm{pot}}$, where $\eta_{N^{+}}^{\mathrm{HS}}$ describes the "hard-sphere" scattering, which is independent of the strength of the potential $U$ at short range and varies smoothly with the energy, and $\eta_{N^{+}}^{\mathrm{pot}}$, which depends on $U$ and gives rise to resonances. Close to the resonances, a strong energy dependence is expected in accordance with the BreitWigner formula. For energies far above the top of the centrifugal barrier, the phase shift decreases and eventually reaches zero, because the importance of the potential becomes negligible and the wave functions approach those of a free particle.

For $N^{+}=5$, the resonances in $\eta_{N^{+}}^{\text {pot }}$ completely dominate the phase evolution below the barrier for both electronic states. In contrast, $\eta_{N^{+}}^{\mathrm{HS}}$ dominates for $N^{+}=11$, with the exception of a narrow resonance for the $X^{+}$state. At a given energy, the phase shift tends to zero as $N^{+}$increases, because the centrifugal term starts to dominate over $U$ in Eq. (2). From the small energy dependence of the phase shifts of the highest partial waves in Fig. 2, one can conclude that a partial-wave expansion of the $\mathrm{D}^{+}+\mathrm{D}$ collision rapidly converges at low energies.

For the classification of resonance features we follow Davis and Thorson [42], who distinguish between shape and orbiting resonances. The former are located energetically below the maximum of the centrifugal barrier of the effective potential and are characterized by a full phase jump of $\pi$. The latter resonances, which may also be regarded as quantum-reflection resonances and include the top-of-barrier and above-barrier resonances in the 

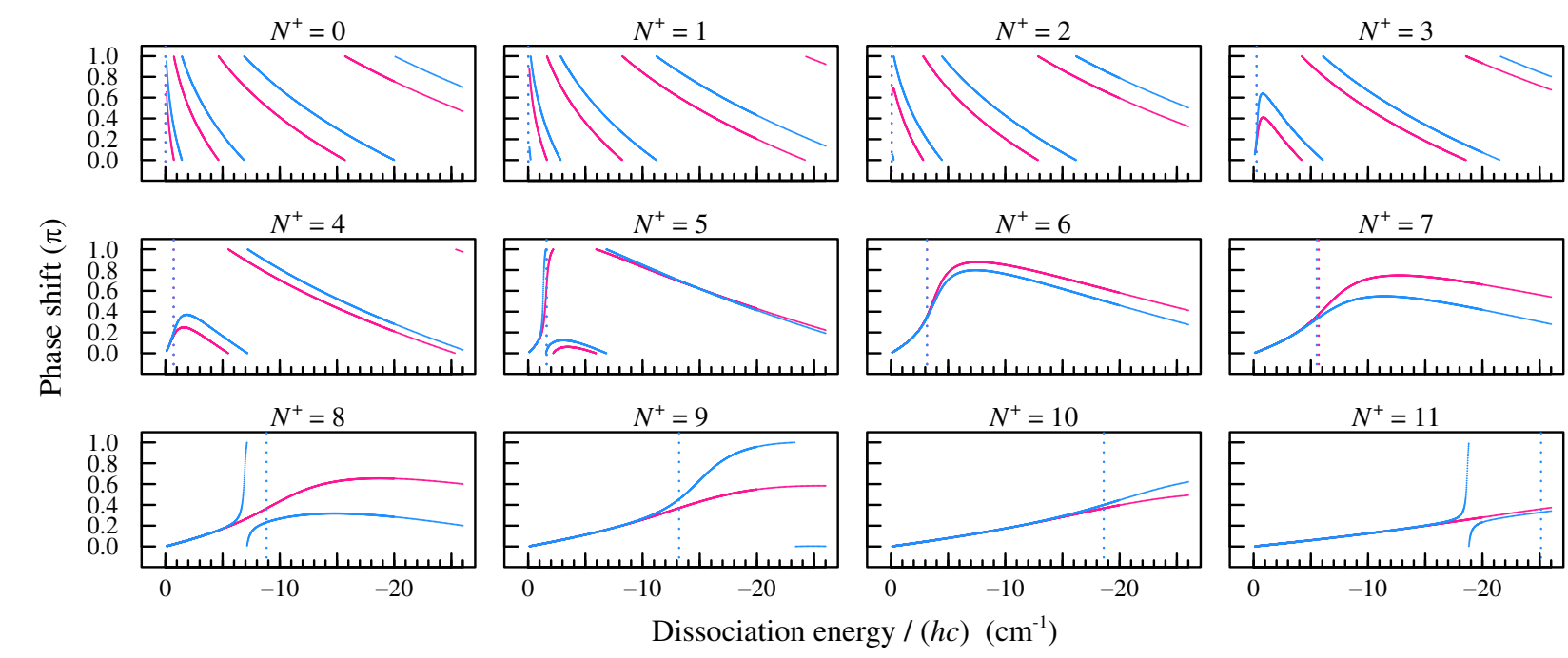

FIG. 2. Energy dependence of the individual partial wave scattering phase shift $\eta_{N^{+}}$(modulo 1 ) of the $\mathrm{D}^{+}+\mathrm{D}$ collision in the $X^{+}$(blue) and $A^{+}$states (red) for the angular-momentum channels $N^{+}=0-11$ at low energies. The dashed lines indicate the positions of the maxima of the centrifugal barriers.

classification of Krstić et al. [27], undergo a phase jump of less than $\pi$. Inspection of Fig. 2 reveals four shape resonances of the $\mathrm{D}^{+}+\mathrm{D}$ collision associated with the $X^{+}$state at low energies $\left[-1.32 \mathrm{~cm}^{-1}\left(N^{+}=5\right)\right.$; $-6.9 \mathrm{~cm}^{-1} \quad\left(N^{+}=8\right) ; \quad-14.9 \mathrm{~cm}^{-1} \quad\left(N^{+}=9\right) ; \quad$ and $\left.-18.6 \mathrm{~cm}^{-1}\left(N^{+}=11\right)\right]$. A fifth shape resonance, associated with the $A^{+}$state, is also observed, as discussed below. Orbiting resonances associated with the $X^{+}\left(N^{+}=3,4,6\right.$, 7, and 10) channels can also be identified in Fig. 2.

To facilitate the identification of resonances, we calculated the relative photoelectron signal intensity of the dissociative-photoionization (DI) in the Franck-Condon approximation as

$$
S(E)=\sum_{N^{+}} c_{N^{+}}\left|\left\langle\phi_{v=12}^{\bar{H}}(R) \mid \phi_{k, N^{+}}^{i}(R)\right\rangle\right|^{2}
$$

using the wave function $\phi_{v=12}^{\bar{H}}(R)$ of the $v=12$ level of the $\bar{H}$ state determined numerically from the potential function of the $H \bar{H}$ state reported by Wolniewicz [43] and energynormalized continuum functions $\phi_{k, N^{+}}^{i}(R)$. The exact calculation of the absolute DI cross section from the $\bar{H}$ state is not possible at present, because no $a b$ initio transition dipole moments are available. The Coulomb approximation, commonly used for calculating transition moments for the excitation of Rydberg states [44,45], cannot be applied in a straightforward manner to the present problem because the ion-pair character of the $\bar{H}$ state implies large $\ell$ mixing in a single-center basis.

The total integrated elastic cross section $\sigma_{\mathrm{el}}$ for the $\mathrm{D}^{+}+$ D collision (see extensive discussion in Refs. [27,46-49]) is given by

$$
\sigma_{\mathrm{el}}=\frac{4 \pi}{k^{2}} \sum_{N^{+}=0}^{\infty}\left[\omega_{X^{+}}^{ \pm} \sin ^{2}\left(\eta_{N^{+}}^{X^{+}}\right)+\omega_{A^{+}}^{ \pm} \sin ^{2}\left(\eta_{N^{+}}^{A^{+}}\right)\right],
$$

where $\omega_{X^{+}}^{ \pm}$and $\omega_{A^{+}}^{ \pm}$are the spin-statistical weights of ortho$\mathrm{D}_{2}{ }^{+}\left[2 / 3\right.$, for even (odd) $N^{+}$channels of the $X^{+}\left(A^{+}\right)$state $]$ and para- $\mathrm{D}_{2}{ }^{+}\left[1 / 3\right.$, for odd (even) $N^{+}$channels of the $X^{+}$ $\left(A^{+}\right)$state].

\section{RESULTS}

The left and right panels of Fig. 3 present the PFI-ZEKE photoelectron spectra of $\mathrm{D}_{2}$ in the region of the dissociative-ionization (DI) threshold recorded via the $\bar{H}(12,4)$ (ortho- $\mathrm{D}_{2}$ ) and $(12,5)\left(\right.$ para- $\left.\mathrm{D}_{2}\right)$ intermediate levels, respectively. In each panel, the spectra are ordered according to the electric-field step causing the field ionization (see Sec. II). The energy scale has been corrected for the field-induced shift of the ionization thresholds. The sharp lines below the DI threshold (i.e., at positive dissociation energies) correspond to weakly bound states of $\mathrm{D}_{2}{ }^{+}$, the $X^{+}\left(25, N^{+}=6,7\right), X^{+}\left(26, N^{+}=0,2,4\right)$ and $A^{+}\left(0, N^{+}=\right.$ $1,3)$ states for ortho- $\mathrm{D}_{2}{ }^{+}$, and the $X^{+}\left(26, N^{+}=1,3\right)$, $A^{+}\left(0, N^{+}=0,2\right)$ and $A^{+}(1,0)$ states for para- ${ }_{2}^{+}$. Several resonances are also observed above the DI threshold (negative dissociation energies) of $\mathrm{D}_{2}{ }^{+}$. Five of these resonances correspond to the $X^{+}\left(25, N^{+}=8\right.$ and 9$), X^{+}$ $(26,5), X^{+}(24,11)$, and $A^{+}(0,5)$ shape resonances mentioned above. Two further resonances, the $X^{+}(25,10)$ and $A^{+}(0,6)$ resonances, can be assigned to orbiting resonances (see also Figs. 2 and 4 below).

The assignment of these resonances was not only made on the basis of the calculations but also using the information contained in the PFI-ZEKE photoelectron spectra 


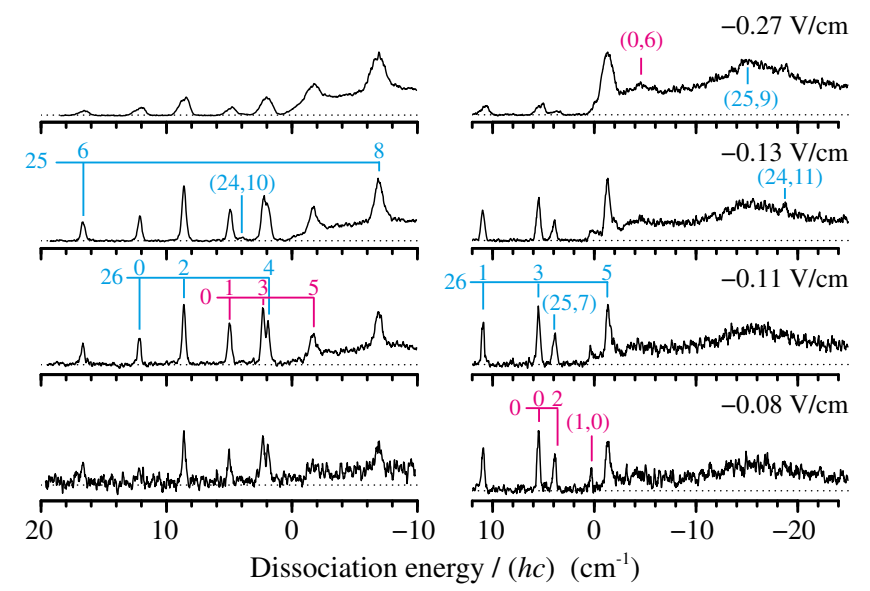

FIG. 3. PFI-ZEKE photoelectron spectra of ortho-D $\mathrm{D}_{2}$ (left) and para- $\mathrm{D}_{2}$ (right) in the vicinity of the DI threshold. The spectra were recorded from the $\bar{H}(12,4)$ and $(12,5)$ intermediate levels, respectively, with the electric field steps $3(-80 \mathrm{mV} / \mathrm{cm}), 5$ $(-110 \mathrm{mV} / \mathrm{cm}), 6(-130 \mathrm{mV} / \mathrm{cm})$, and $8(-270 \mathrm{mV} / \mathrm{cm})$ of the pulse sequence described in Sec. II (from bottom to top). The energy scale was corrected for the field-induced shift of the ionization thresholds. See text for details.

recorded from the $N=0$ to 6 rotational levels of the $\bar{H}(12)$ intermediate state depicted in Fig. 4. These spectra are dominated, up to about $25 \mathrm{~cm}^{-1}$ above the DI threshold (left panels), by the shape and orbiting resonances of $\mathrm{D}_{2}{ }^{+}$ mentioned above, the relative intensities of which rapidly vary with $N$. The ion-pair character of the $\bar{H}$ state enables one to observe ionizing transitions associated with changes $\Delta N\left(=N^{+}-N\right)$ of the rotational quantum numbers up to 6, as explained in Ref. [29]. Consequently, transitions to shape resonances with $N^{+} \geq 8$ for ortho- $\mathrm{D}_{2}{ }^{+}$(or 9 for para $\left.\mathrm{D}_{2}{ }^{+}\right)$are not observable from $N=0(N=1)$ but gain in importance as $N$ increases.

At higher energies (right panels in Fig. 4), the spectra all consist of the same slow and irregular oscillations of the photoelectron signal, which primarily reflect the FranckCondon factors for dissociative ionization from the $\bar{H}(12)$ state. This aspect is illustrated in the top right panel of the figure, which displays, as green trace, the photoelectron signal we obtain by adding the Franck-Condon factors calculated for the transitions from the $\bar{H}(12,0)$ level to the $N^{+}=0,2$, and 4 channels weighted by factors of $1,0.3$, and 0.15 , respectively. The intensity distribution calculated in this way reproduces the minima and maxima observed experimentally well. A perfect agreement cannot be expected because the Franck-Condon approximation becomes inaccurate over the broad range of internuclear distances covered by the $\bar{H}(12)$ state.

The positions and widths (FWHM) of all shape resonances observed in these spectra are listed in Table I, where they are compared with the values calculated as explained in Sec. III. They were determined by first subtracting the slowly varying nonresonant photoelectron signal, and then
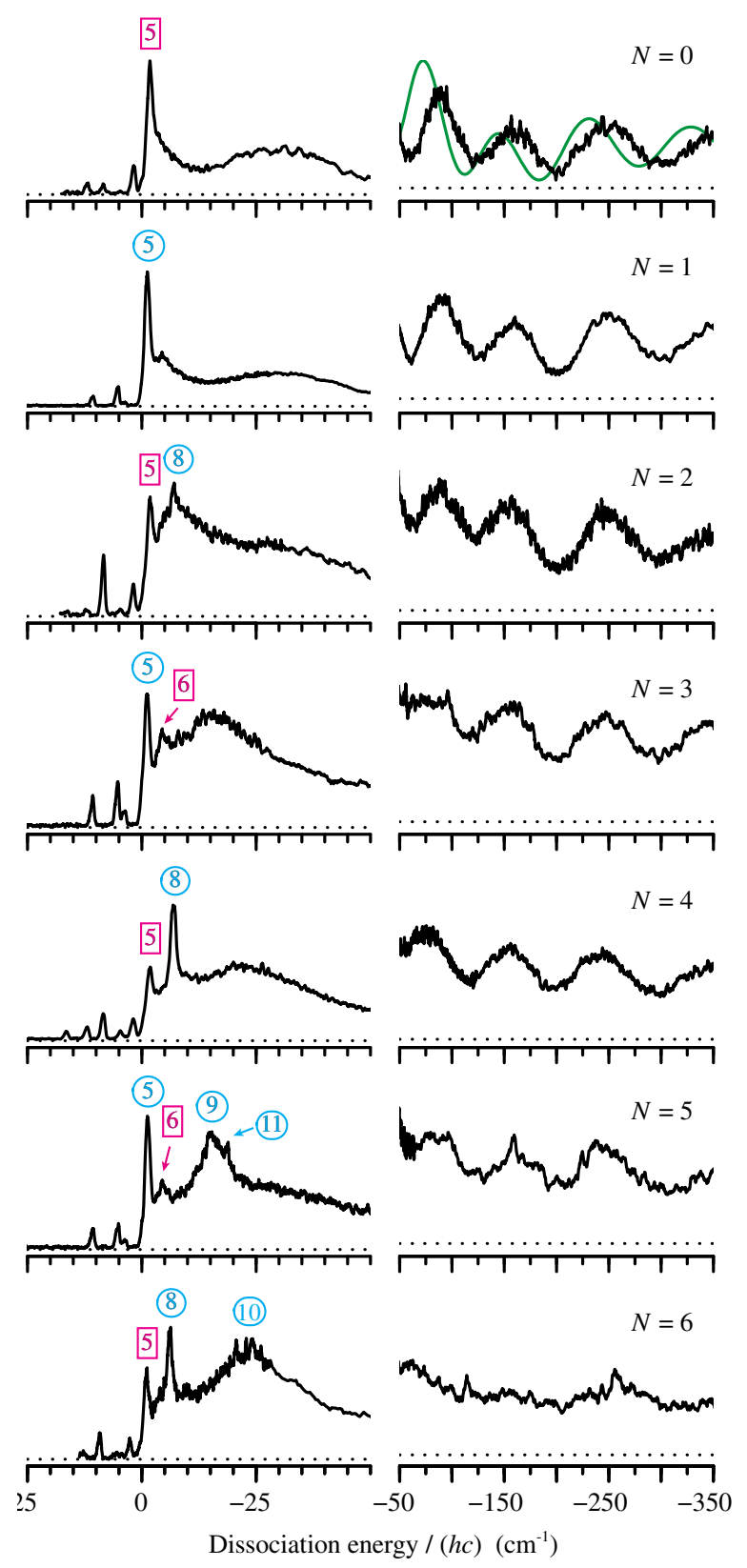

FIG. 4. PFI-ZEKE photoelectron spectra of $\mathrm{D}_{2}$ recorded with pulse $9(-670 \mathrm{mV} / \mathrm{cm})$ of the field-ionization sequence from the $N=0-6$ rotational levels of the $\bar{H}(v=12)$ state. The resonances associated with the $X^{+}$and $A^{+}$states are labeled with the value of $N^{+}$surrounded by circles and squares, respectively. The left panels show the region where the shape resonances dominate and the right panels present the region where the spectra are dominated by the slow oscillations of the Franck-Condon factors on an expanded vertical scale $(\times 2)$ and reduced horizontal scale $(\times 0.25)$. The green trace in the top right panel corresponds to calculated Franck-Condon factors (see text). The dotted lines give the zero-signal level.

fitting Voigt profiles assuming a Gaussian instrumental function with FWHM given by the linewidths of the transitions to the bound levels. The calculated and measured positions of all resonances agree within the 
experimental uncertainties. Their widths also agree well, with the exception of the $X^{+}(25,8)$ shape resonance, which appears broader in the experimental spectrum. This discrepancy and a similar discrepancy observed for the $X^{+}(17,7)$ shape resonance of $\mathrm{H}_{2}^{+}[28]$ are both too large to be explained by the experimental uncertainties. The analysis of the widths of these two resonances in the PFIZEKE photoelectron spectra recorded for the different field steps of the field-ionization sequence further reveals that the broadening is independent of the values of $n$ probed by these field steps, an observation we shall return to in the discussion of the broadening mechanism in Sec. VA.

Although the $\mathrm{D}^{+}+\mathrm{D}(n \geq 2)$ channels are strongly closed at the low energies studied here, their effects on the resonance positions and widths are noticeable at our resolution. Indeed, the good agreement between calculated and experimental values of the resonance positions and widths can only be reached when nonadiabatic interactions are included in the calculations. Nonadiabatic effects are even more pronounced in the $\mathrm{H}^{+}+\mathrm{H}$ system (see Refs. [28,29]).

Figure 5 depicts the effective potentials $U+$ $\left\{\left[N^{+}\left(N^{+}+1\right)\right] /\left(2 \mu_{\text {rot }} R^{2}\right)\right\}$ of the $X^{+}$state for $N^{+}=0$, 5,10 (blue traces) and of the $A^{+}$state for $N^{+}=0,5$ (red traces) together with the wave functions of the resonances at the resonance centers. The resonances represent the

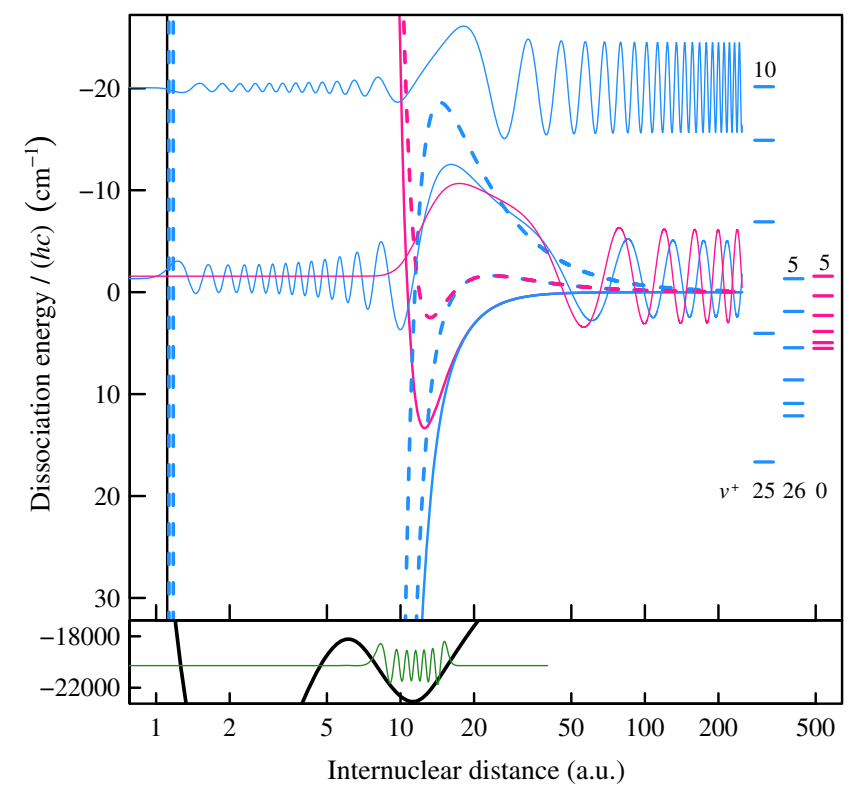

FIG. 5. Top panel: Effective potential $U+\left\{\left[N^{+}\left(N^{+}+1\right)\right] /\right.$ $\left.\left(2 \mu_{\text {rot }} R^{2}\right)\right\}$ of the $X^{+}$state of $\mathrm{D}_{2}^{+}$for $N^{+}=5$ and 10 (blue) and of the $A^{+}$state for $N^{+}=5$ (red) together with the wave functions of the $X^{+}(26,5)$ and $(25,10)$ and the $A^{+}(0,5)$ resonances at the resonance centers. The rotationless $\left(N^{+}=0\right)$ potentials $U$ are indicated as solid lines. The energies of bound states and resonances having the same vibrational quantum numbers are indicated on the right side. Bottom panel: Potential function of the $H \bar{H}^{1} \Sigma_{g}^{+}$state and wave function of the $\bar{v}=12$ level of $\mathrm{D}_{2}$. continuation of the rotational progressions of specific vibrational levels through the DI threshold, as indicated on the right of the figure. The wave functions at the center of the resonances are characterized by a large and slowly varying amplitude in the range between 7 and $17 a_{0}$, corresponding to the range where the intermediate $\bar{H}(v=12)$ nuclear wave function is different from zero (see bottom panel of Fig. 5).

In contrast to $\mathrm{H}_{2}{ }^{+}$, which does not have any shape resonances associated with the $A^{+}$state, $\mathrm{D}_{2}{ }^{+}$exhibits one such resonance, the $A^{+}(0,5)$ resonance, which is clearly observable in the PFI-ZEKE photoelectron spectra of ortho- $\mathrm{D}_{2}$ (see Figs. 3 and 4). The observation of narrow shape resonances in both the $X^{+}$and $A^{+}$states of $\mathrm{D}_{2}{ }^{+}$is relevant for the low-temperature radiative association of $D^{+}$and $\mathrm{D}$ forming $\mathrm{D}_{2}{ }^{+}$and for the $\mathrm{D}^{+}+\mathrm{D}$ collision cross sections, as discussed further in Secs. V B and V C.

\section{DISCUSSION}

Our approach to study the $\mathrm{D}^{+}+\mathrm{D}$ collision consists of observing spectra of the dissociating $\mathrm{D}_{2}{ }^{+}$ion core within the orbit of a highly excited $(n \gg 200)$ Rydberg electron. In this section, we discuss important aspects of this approach in more detail. In Sec. VA, we analyze the possible influences of the Rydberg electron on the ion-core dissociation and of the ion-core dissociation on the Rydberg electron. Specifically, we show that the former is negligible within the precision and accuracy limits of our experiments, but that the latter can be significant and actually is the cause of the artificial broadening of the $X^{+}(25,8)$ shape resonance of $\mathrm{D}_{2}{ }^{+}$(see Table I) and of the $X^{+}(17,7)$ shape resonance of $\mathrm{H}_{2}{ }^{+}$reported in Ref. [28]. In Sec. V B, we show that our half-collision approach to study the $\mathrm{H}^{+}+\mathrm{H}$ and $\mathrm{D}^{+}+\mathrm{D}$ collision system provides access to aspects of these collisions, in particular the elastic-scattering cross sections, that would be extremely difficult to observe in scattering experiments. Finally, in Sec. V C, we derive, from our results, the cross sections and thermal rate constants for the $\mathrm{H}^{+}+\mathrm{H} \rightarrow \mathrm{H}_{2}^{+}$and $\mathrm{D}^{+}+\mathrm{D} \rightarrow \mathrm{D}_{2}{ }^{+}$ radiative-association reactions.

\section{A. Effect of the charge localization in the dissociating ion core on the Rydberg electron}

The analysis of the spectral structures observed in the PFI-ZEKE photoelectron spectra presented in Sec. IV disregards a possible influence of the Rydberg electron on the $\mathrm{D}_{2}{ }^{+}$dissociation dynamics. The Rydberg states that contribute to the spectra we used to determine the resonance widths and positions have $n$ values beyond 270 and classical radii $a_{0} n^{2} \geq 3.8 \mu \mathrm{m}$. Under our experimental conditions, these Rydberg states are fully $\ell$ mixed by the stray electric fields present in the photoexcitation region $[50,51]$, so that one can safely assume that the Rydberg electron does not significantly penetrate in the $\mathrm{D}_{2}{ }^{+}$core 
TABLE I. Experimental (exp) and calculated (calc) positions and widths (in $\mathrm{cm}^{-1}$ ) of the observed shape resonances of $\mathrm{D}_{2}{ }^{+}$and $\mathrm{H}_{2}{ }^{+}$ (last two lines) and corresponding asymptotic fragment velocities $v$ (in $\mathrm{m} / \mathrm{s}$ ). $\Gamma_{\mathrm{b}}$ represents the contribution to the experimental width originating from the mechanism discussed in Sec. VA. The experimental uncertainties represent one standard deviation.

\begin{tabular}{|c|c|c|c|c|c|c|}
\hline Resonance & $\tilde{\nu}_{\exp }$ & $\Gamma_{\exp }$ & $\tilde{\nu}_{\text {calc }}$ & $\Gamma_{\text {calc }}$ & $v$ & $\Gamma_{\mathrm{b}}{ }^{\mathrm{a}}$ \\
\hline$X^{+}(26,5)$ & $-1.336(16)$ & $0.19(9)$ & -1.32 & 0.16 & 88 & 0.13 \\
\hline$X^{+}(25,8)$ & $-6.915(19)$ & $0.47(9)$ & -6.90 & 0.31 & 203 & 0.29 \\
\hline$X^{+}(25,9)$ & $-16.2(15)$ & $6.7(8)$ & -14.90 & 5.73 & 298 & 0.43 \\
\hline$X^{+}(25,10)^{\mathrm{b}}$ & $-24(6)$ & $\ldots$ & -20.2 & $\cdots$ & $\cdots$ & $\ldots$ \\
\hline$X^{+}(24,11)$ & $-18.75(11)$ & $\leq 0.6$ & -18.63 & 0.24 & 333 & 0.48 \\
\hline$A^{+}(0,5)$ & $-1.685(17)$ & $0.41(12)$ & -1.59 & 0.36 & 97 & 0.16 \\
\hline$A^{+}(0,6)^{\mathrm{b}}$ & $-4(1)$ & $\ldots$ & -3.69 & $\ldots$ & $\ldots$ & $\ldots$ \\
\hline$X^{+}(18,4)^{\mathrm{c}}$ & $-1.84(4)$ & $0.21(7)$ & -1.88 & 0.194 & 150 & 0.21 \\
\hline$X^{+}(17,7)^{\mathrm{c}}$ & $-11.08(6)$ & $0.56(8)$ & -11.10 & 0.163 & 364 & 0.53 \\
\hline
\end{tabular}

${ }^{\mathrm{a}}$ Contribution to the experimental width from $n$ changes caused by charge localization during dissociation, see text for details.

${ }^{\mathrm{b}}$ Orbiting resonance.

${ }^{\mathrm{c}} \mathrm{H}_{2}{ }^{+}$resonance; data from Ref. [28].

region for the internuclear distances relevant for the description of the shape resonances, i.e., for $R \leq 50 \AA$. The electric field of an electron at a distance of $3.8 \mu \mathrm{m}$ is only $1 \mathrm{~V} / \mathrm{cm}$ and its effect on the ion core is negligible. One can, therefore, safely conclude that the Rydberg electron does not significantly affect the positions nor the widths of the $\mathrm{D}_{2}{ }^{+}$shape resonances but only acts as a spectator during the core-dissociation process.

However, one cannot conclude that the core dissociation does not affect the Rydberg electron. Experimentally, we have verified that the field-ionization signal observed above the core-dissociation threshold stems from the field ionization of D-atom Rydberg states, which implies a localization of the ion-core charge on one of the two fragments between photoexcitation and pulsed-field ionization. This localization represents a displacement of the center of charge from the center of mass of the dissociating $\mathrm{D}_{2}{ }^{+}$core to one of the two fragments, as depicted schematically in Fig. 6 for the two extreme situations corresponding to a localization of the core charge on the fragment located closest to [panel (b)], or furthest away from [panel (c)], the "semiclassical" Rydberg electron. In these two situations, the effect is an abrupt change of the classical radius by $\pm v_{\mathrm{D}} t_{\mathrm{loc}}$, where $v_{\mathrm{D}}$ is the velocity of the fragments in the center-of-mass frame and $t_{\mathrm{loc}}$ is the time at which charge localization takes place. The resulting change $\Delta n=n^{\prime}-n$ of the principal quantum number of the electron can be obtained from Eq. (6)

$$
a_{0} n^{\prime 2}=a_{0} n^{2} \pm v_{\mathrm{D}} t_{\mathrm{loc}}
$$

and is accompanied by a change of the electron binding energy $\Delta E_{n n^{\prime}}$ given by

$$
\frac{\Delta E_{n n^{\prime}}}{h c}=\operatorname{Ry}\left(\frac{1}{n^{\prime 2}}-\frac{1}{n^{2}}\right)
$$

which results in a broadening of the shape resonances in the PFI-ZEKE photoelectron spectrum by $2 \Delta E_{n n^{\prime}}$. In Eq. (7), we

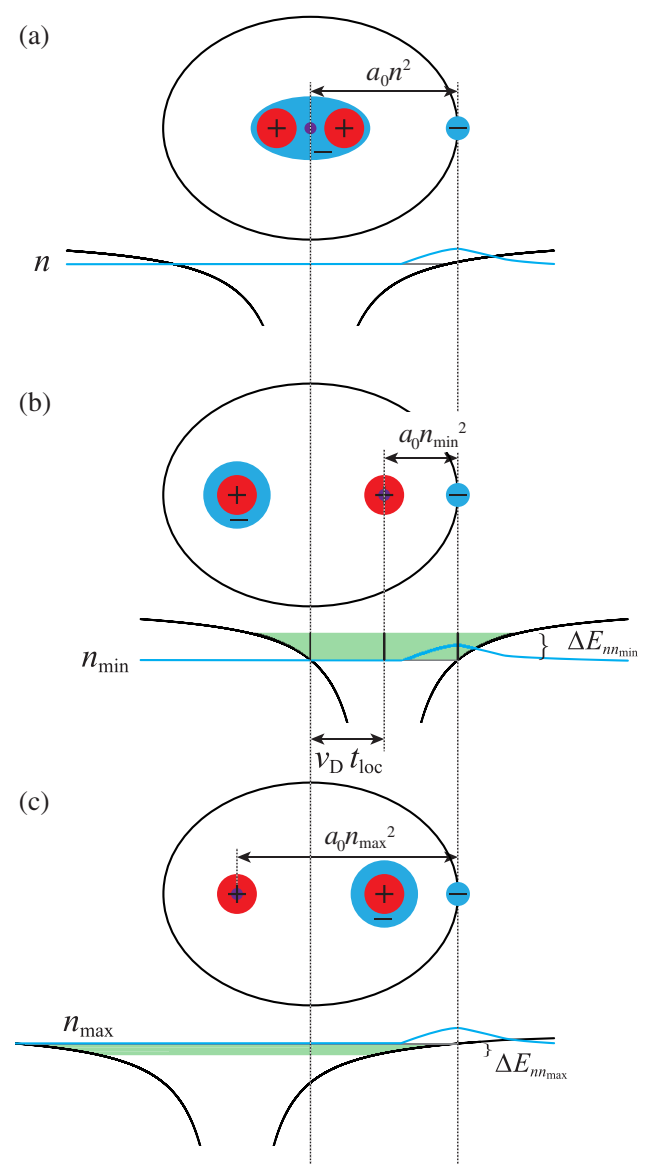

FIG. 6. Schematic representation of the effect, on a high- $n$ Rydberg electron, of the charge localization of the dissociating $\mathrm{D}_{2}{ }^{+}$ion core on one of the two fragments. The situations prior to charge localization, and after localization of the core charge on the fragment located closest to, and furthest away from, the "semiclassical" Rydberg electron, are depicted in panels (a), (b), and (c), respectively. 
make the approximation that the Rydberg constants (Ry) for $\mathrm{D}$ and $\mathrm{D}_{2}$ are the same. This approximation is excellent and the different values of the Rydberg constant for $\mathrm{D}$ and $\mathrm{D}_{2}$ do not cause a significant redistribution of $n$ nor a measurable broadening of the shape resonances.

Numerous effects may be responsible for the charge localization, such as inhomogeneous electric fields, the interaction with neutral atoms and molecules of the background gas, the coupling between the $A^{+}$and $X^{+}$states mediated by the hyperfine interaction, or a Fermi-contactlike interaction of the Rydberg electron with the dissociating $\mathrm{D}_{2}{ }^{+}$ion core. Under our experimental conditions, we expect this last interaction to be dominant.

A theoretical explanation for the observed behavior involves the consideration of the individual terms of the complete nonrelativistic Hamiltonian $\hat{H}_{\text {TOT }}$ of the neutral molecule $\left(\mathrm{H}_{2}\right.$ or $\left.\mathrm{D}_{2}\right)$ for the case where one electron is in a highly excited Rydberg state

$$
\hat{H}_{\mathrm{TOT}}=\hat{H}_{\mathrm{ion}}+\hat{H}_{\mathrm{Rg}}+\hat{H}_{\mathrm{res}} .
$$

In Eq. (8) $\hat{H}_{\text {ion }}$ is the full Hamiltonian of $\mathrm{H}_{2}{ }^{+}$or $\mathrm{D}_{2}{ }^{+}, \hat{H}_{\mathrm{Rg}}$ contains the kinetic-energy operator of the Rydberg electron and the Coulomb potential arising from the ion-core charge, and the residual term $\hat{H}_{\text {res }}$, consists of the electronelectron repulsion term, the nonspherically symmetric part of the ion-core-Rydberg-electron potential, and the nonadiabatic corrections involving the two electrons. $\hat{H}_{\text {res }}$ is the term responsible for the observed charge localization in the dissociating ion core. At very high $n$ values, where $R \ll a_{0} n^{2}, \hat{H}_{\mathrm{Rg}}$ is given by Rydberg's formula and the interaction $\hat{H}_{\text {res }}$ is very weak and restricted to the ion-core region. $\hat{H}_{\text {res }}$ is thus describable by a Fermi-contact term. Such a term is proportional to the probability density of the Rydberg electron in the core region. For the fully $\ell$-mixed Stark state detected in our PFI-ZEKE-PE spectra, this density scales as $n^{-4}$ [50-52], which would imply that the charge localization time (or core-decoherence time) is proportional to $n^{4}$, i.e.,

$$
t_{\mathrm{loc}}=t_{\mathrm{loc}}^{*} n^{4}
$$

Combining Eqs. (6)-(9) leads to the conclusion that the broadening $\Gamma_{b}$ of the resonances by the charge-localization on one of the fragments is given by

$$
\Gamma_{b}=2 \frac{\Delta E_{n n^{\prime}}}{h c}=2 \frac{\operatorname{Ry} v_{\mathrm{D}} t_{\mathrm{loc}}}{a_{0}\left(n^{\prime} n\right)^{2}} \approx 2 \frac{\operatorname{Ry} v_{\mathrm{D}} t_{\mathrm{loc}}^{*}}{a_{0}}
$$

and is independent of $n$ to first order, which is consistent with the experimental observations (see Sec. IV). Moreover, the broadening only depends on the mass of the fragment through the dependence of the fragment velocity on the resonance energy $\left(v_{\mathrm{D}}=\sqrt{\left|E_{\mathrm{diss}}\right| / m}\right)$. Consequently, $t_{\text {loc }}^{*}$ should be the same for $\mathrm{H}_{2}$ and $\mathrm{D}_{2}$.

Attributing the broadening of the $X^{+}(17,7)$ shape resonance of $\mathrm{H}_{2}$ to this mechanism, we obtain a value of $3.55 \times 10^{-19} \mathrm{~s}$ for $t_{\mathrm{loc}}^{*}$, which translates to a charge-localization time of $1.9 \mathrm{~ns}$ at $n=270$. With this value of $t_{\mathrm{loc}}^{*}, \Gamma_{b}$ can be calculated for all shape resonances observed experimentally and the results are presented in the last column of Table I. The effect of the broadening is negligible for resonances located close to threshold and for broad resonances. The only two widths to be significantly affected are those of the $X^{+}(17,7)$ resonance of $\mathrm{H}_{2}{ }^{+}$ and the $X^{+}(25,8)$ of $\mathrm{D}_{2}{ }^{+}$. Assuming that the different contributions to the widths observed in the PFI-ZEKE photoelectron spectra add in quadrature, one obtains a corrected width of $0.37(9) \mathrm{cm}^{-1}$ for the $X^{+}(25,8)$ shape resonance of $\mathrm{D}_{2}{ }^{+}$, which is in agreement with the width $\left(0.31 \mathrm{~cm}^{-1}\right)$ calculated $a b$ initio (see Table I).

The simple model presented here to estimate the effects on the Rydberg electron of the charge localization in the dissociating core provides an explanation for all observations: It accounts for the fact that only two widths (out of seven) of the observed shape resonances measured by PFIZEKE photoelectron spectroscopy differ from the $a b$ initio predictions. It provides an explanation for the fact that, in these two cases, the broadening is independent of $n$. Finally, the broadening observed for the $X^{+}(17,7)$ resonance of $\mathrm{H}_{2}{ }^{+}$can be used to estimate the broadening of the $X^{+}(25,8)$ shape resonance of $\mathrm{D}_{2}{ }^{+}$without adjustment of any parameter. To our knowledge, this effect has not been discussed yet in the literature.

\section{B. $\mathrm{D}^{+}+\mathrm{D}$ scattering cross sections}

The $\mathrm{D}^{+}+\mathrm{D}$ and $\mathrm{H}^{+}+\mathrm{H}$ collisions are the prototypical examples of collisions of identical bosons and fermions, respectively. Consequently, many theoretical studies have discussed them in detail [27,46-49]. The observation of cross sections for such collisions at low temperature has not been possible so far and is generally believed to be impossible in traditional scattering experiments [27]. The only experimental cross sections at low energy available so far concern the $\mathrm{H}^{+}+\mathrm{D}$ and $\mathrm{D}^{+}+\mathrm{H}$ reactions at collisions energies corresponding to temperatures beyond $100 \mathrm{~K}$ [53]. In collision experiments, difficulties arise from the contributions from a multitude of partial waves associated with both the $X^{+}$and $A^{+}$states. Moreover, experiments involving spin-polarized nuclei, needed to study the effects resulting from the (in)distinguishability of the nuclei, are beyond reach at low temperatures (see, e.g., discussion in Ref. [27]).

The experimental study presented in Sec. IV represents a half-collision approach to characterize the $\mathrm{D}^{+}+\mathrm{D}$ collision. A key advantage of this approach is the possibility to study ortho- and para- $\mathrm{D}_{2}{ }^{+}$separately. The ortho and para 
selection of the collision complex is, however, not equivalent to the use of spin-polarized deuterons and $\mathrm{D}$ atoms in a collision experiment because ortho- and para- $\mathrm{D}_{2}{ }^{+}$consist of a mixture of the two hyperfine components $(F=1 / 2,3 / 2)$ of the $\mathrm{D}(1 s)$ atom. By using a resonant multiphoton excitation scheme through a given rotational level $(N)$ of the $\bar{H}^{1} \Sigma_{g}^{+}(v=12)$ state [see Eq. (1)], we (i) prepare the $\mathrm{D}^{+}+\mathrm{D}$ collision complex at a well-defined energy and in a well-defined nuclear-spin state (para, $I=1$, or ortho $I=0,2$ ) within the orbit of the (noninteracting) Rydberg electron, and (ii) restrict the number of partial waves in the half collisions through rovibronic photoionization selection rules [54] for an odd- $\ell$ photoelectron partial wave (see Sec. IV), as summarized in Table II.

The advantages of the half-collision approach are best illustrated with the example of the $N^{+}=5$ partial wave. The two narrow $X^{+}\left(N^{+}=5\right)$ and $A^{+}\left(N^{+}=5\right)$ shape resonances are almost degenerate (see Fig. 7) and would be extremely difficult to resolve in a measurement of the total elastic cross section. They are, however, easily distinguished in the PFI-ZEKE photoelectron spectra because the $X^{+}\left(N^{+}=5\right)$ resonance appears in the photoelectron spectra of para- $\mathrm{D}_{2}$, and the $A^{+}\left(N^{+}=5\right)$ resonance in the spectra of ortho- $\mathrm{D}_{2}$ (see Fig. 4).

By identifying the features associated with individual partial waves, e.g., the widths and positions of individual shape resonances, and reproducing these features in $a b$ initio calculations, we validate the calculated elastic-scattering phase shifts $\eta_{N^{+}}^{X^{+}}(E)$ and $\eta_{N^{+}}^{A^{+}}(E)$ (see Fig. 2). These phase shifts are all that is needed to calculate the elastic-scattering cross sections [see Eq. (5)] and it is instructive to compare these cross sections with the Franck-Condon factors for DI calculated with Eq. (4) (see Fig. 7).

The bound-continuum Franck-Condon factors for accessing the individual $N^{+}$channels of the $X^{+}$and $A^{+}$ states from the $\bar{H}(v=12)$ state are depicted as blue and red traces in Fig. 7. The low-energy onset of the DI cross section gradually shifts to higher energies with increasing value of $N^{+}$following a $E^{N^{+}+1 / 2}$ threshold law. The Franck-Condon factors for partial waves not affected by resonances (e.g., $N^{+}=0$ and 1) are small and show slow oscillations reflecting the projection of the $\bar{H}(12)$ nuclear wave function onto the repulsive part of the $X^{+}$and $A^{+}$ potentials. Close to the $X^{+}\left(N^{+}=5,8,9,10\right)$ and $A^{+}\left(N^{+}=5\right)$ shape resonances, the Franck-Condon factors

TABLE II. Selection of specific partial waves of the half collision of ortho- and para- $\mathrm{D}_{2}{ }^{+}$by carrying out the multiphoton excitation sequence through selected rotational levels $(N)$ of the $\bar{H}^{1} \Sigma_{g}^{+}(v=12)$ state.

\begin{tabular}{llc}
\hline \hline Ionic state & $\bar{H}($ Neven $) ; I=1$ & $\bar{H}($ Nodd $) ; I=0,2$ \\
\hline$X^{+}$ & $N^{+}=0,2,4, \ldots$ & $N^{+}=1,3,5, \ldots$ \\
$A^{+}$ & $N^{+}=1,3,5, \ldots$ & $N^{+}=0,2,4, \ldots$ \\
\hline \hline
\end{tabular}

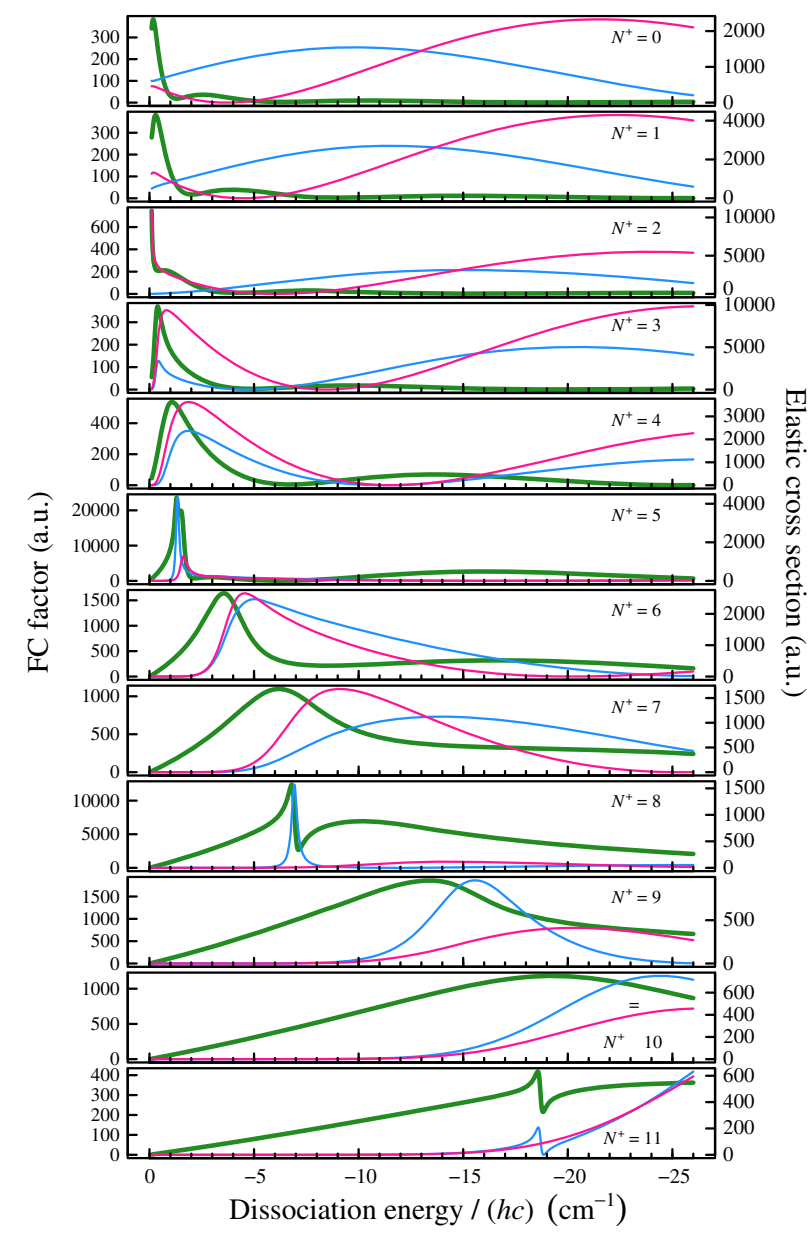

FIG. 7. Bound-continuum Franck-Condon (FC) factors for transitions from the $\bar{H}(v=12)$ state to the $\mathrm{D}^{+}+\mathrm{D}$ dissociation channels with angular-momentum quantum numbers $N^{+}=0-9$, respectively. The solid blue and red lines correspond to the $X^{+}$ and $A^{+}$channels, respectively. The total elastic cross section for the individual partial waves are given by the solid green lines. Note the different vertical scales.

are dominated by sharp spectral structures. The line shapes are often symmetric but can also appear as Fano profiles, as is the case for the $X^{+}\left(N^{+}=11\right)$ shape resonance. The orbiting resonances display a different behavior (see, e.g., $N^{+}=6$ ). They enhance the Franck-Condon factors because of the larger amplitude of the continuum wave functions at smaller internuclear distances. The resulting spectral features are strongly asymmetric and cannot be parametrized using conventional line-shape models, so that the specification of a linewidth becomes problematic.

The green traces in Fig. 7 depict the contributions of the individual partial waves to the elastic cross section calculated with Eq. (5). The low-energy onset of $\sigma_{\text {el }}$ gradually shifts to higher energies with increasing value of $N^{+}$as $\sigma_{\mathrm{el}} \propto E^{\left(2 N^{+}+1\right)}=\left(E^{\left(N^{+}+1 / 2\right)}\right)^{2}$, as expected from the Wigner threshold law [55]. Compared to the DI cross sections, the factor $E^{N^{+}+1 / 2}$ enters twice in $\sigma_{\text {el }}$ because the 
centrifugal barrier has to be traversed twice, once to form the collision complex, once when the complex dissociates. Although the positions and widths of the shape resonances are identical in the Franck-Condon factors $S(E)$ and the elastic cross section $\sigma_{\mathrm{el}}$, the actual line shapes can be different. For example, the $X^{+}\left(N^{+}=8\right)$ shape resonance appears as a Fano profile in $\sigma_{\text {el }}$ because of the background phase shift at the position of the resonance, but as a Lorentzian line in $S(E)$. For partial waves not affected by resonances, the Franck-Condon factors obviously differ from $\sigma_{\mathrm{el}}$. Moreover, the intensity maxima of broad orbiting resonances are typically observed at slightly lower energies in $\sigma_{\mathrm{el}}$ than in $S(E)$. This behavior can be explained by the nodal structure of the $\bar{H}(12)$ nuclear wave function.

\section{C. $\mathrm{D}^{+}+\mathrm{D}$ radiative association cross sections at low temperatures}

The observation of a relatively narrow shape resonance of the $A^{+}$state $\left[A^{+}(0,5)\right.$, located $1.68 \mathrm{~cm}^{-1}$ above the $\mathrm{D}^{+}+\mathrm{D}$ dissociation threshold] is relevant for the lowtemperature radiative-association dynamics. The resonance can radiate to any $\left(v^{+}, N^{+}=4\right.$ or 6$)$ bound level of the $X^{+}$ state of $\mathrm{D}_{2}{ }^{+}$via electric-dipole-allowed transitions. The $X^{+}(26,5)$ resonance is also expected to be relevant because it can radiate to the $A^{+}(0,4)$ bound level of $\mathrm{D}_{2}^{+}$, which can radiate further to bound levels of the $X^{+}$state. In this subsection, we analyze the role of the shape resonances in $\mathrm{D}_{2}{ }^{+}$and $\mathrm{H}_{2}{ }^{+}$in the corresponding radiative associations following earlier treatments $[7,8,56-58]$.

To treat the formation of a diatomic molecule by radiative association of two colliding atoms [in the present case $\mathrm{D}^{+}$and $\left.\mathrm{D}(1 s)\right]$, one considers the two atoms approaching each other in an initial partial wave of angular-momentum quantum number $N^{\prime}$ in the dissociation continuum of a given electronic state $i$ and at a collision energy $E_{\text {coll }}=k^{2} /\left(2 \mu_{\text {vib }}\right)$, where $k$ is the asymptotic wave number of the collision (for clarity, we omit the label + to designate the ionic quantum numbers in this section). The partial cross section for a radiative transition to a bound rovibrational state $\left(v^{\prime \prime}, N^{\prime \prime}\right)$ of the electronic state $f$ with a binding energy $E_{v^{\prime \prime}, N^{\prime \prime}}$ is given in the electric-dipole approximation by $[8,57,58]$

$$
\begin{aligned}
& \sigma\left(f, v^{\prime \prime}, N^{\prime \prime} ; i, k, N^{\prime}\right) \\
& \quad=\frac{4 \pi^{2} \alpha^{3}}{3 \mu E}\left(E_{\text {coll }}-E_{v^{\prime \prime}, N^{\prime \prime}}\right)^{3} S_{N^{\prime \prime}, N^{\prime}}\left|M_{N^{\prime \prime}, N^{\prime}}^{f i}\right|^{2},
\end{aligned}
$$

where $S_{N^{\prime \prime}, N^{\prime}}$ is the Hönl-London factor and $M_{N^{\prime \prime}, N^{\prime}}^{f i}$ is the vibronic transition moment, which we calculated in the adiabatic approximation. Because the electron spin is not affected by electric-dipole transition and the spin-orbit coupling is insignificant in the $X^{+}$and $A^{+}$states of $\mathrm{H}_{2}{ }^{+}$ and $\mathrm{D}_{2}{ }^{+}$, the degeneracy factor $g_{\mathrm{es}}$ cancels out when normalizing the spontaneous emission rate per particle and does therefore not appear in Eq. (11).
The collision energy $E_{\text {coll }}$ (positive) and the binding energy $E_{v^{\prime \prime}, N^{\prime \prime}}$ (negative) are both defined with respect to $U(R=\infty)$, so that the energy of the emitted photon is given by $E_{\mathrm{ph}}=E_{\text {coll }}-E_{v^{\prime \prime}, N^{\prime \prime}}$. In the case of $\mathrm{D}_{2}{ }^{+}$the $A^{+2} \Sigma_{u}^{+}-X^{+2} \Sigma_{g}^{+}$charge-transfer transition moment lies parallel to the internuclear axis and behaves at large $R$ values as $\sim R / 2$ [59]. The transition dipole-moment function $D_{f i}(R)$ was calculated using the BO electronic wave functions obtained in Ref. [29], as outlined in Ref. [60].

The total radiative-association cross section $\sigma_{i}$ for a collision in the electronic state $i$ is obtained by summing over all allowed transitions between the partial waves $N^{\prime}$ and the final states $\left(v^{\prime \prime}, N^{\prime \prime}\right)$, weighted by the probability $p_{N^{\prime}, i}$ for an approach of the two particles in the electronic state $i$. In the case of the radiative associations $\mathrm{H}(1 s)+\mathrm{H}^{+}$ and $\mathrm{D}(1 s)+\mathrm{D}^{+}$, the sum over $N^{\prime \prime}$ is restricted to $R$-branch $\left(N^{\prime}=N^{\prime \prime}+1\right)$ and $P$-branch $\left(N^{\prime}=N^{\prime \prime}-1\right)$ transitions by the $\Delta N= \pm 1$ selection rule for $\Sigma-\Sigma$ transition. The contributions from the electron spin $S$ were neglected, so that $J=N$ and $\Delta S=0$.

If the two nuclei are distinguishable, $p_{N^{\prime}, i}$ is independent of $N^{\prime}$. In the cases of $\mathrm{D}+\mathrm{D}^{+}$and $\mathrm{H}+\mathrm{H}^{+}, p_{N^{\prime}, i}$ would be equal to $1 / 2$ because the approaches of the two atoms in the $\mathrm{X}^{+}$and $\mathrm{A}^{+}$states are equally likely. However, in both cases, the nuclei are indistinguishable and it is necessary to consider the restrictions imposed by the Pauli principle. For two identical nuclei with nuclear spin $I_{1}=I_{2}$, the total wave function must be either symmetric $(s)$ or antisymmetric (a) with respect to the permutation of the nuclear coordinates if $I_{1}$ is integer [as is the case for D $\left(I_{1}=1\right)$ ] or half-integer [as is the case for $\mathrm{H}\left(I_{1}=1 / 2\right)$ ], respectively. The probabilities of approach in a given electronic state thus become dependent on $N^{\prime}$ and are given by the spinstatistical weights of the molecular wave functions, as summarized in Table III.

The radiative-association cross sections for the formation of $\mathrm{D}_{2}{ }^{+}$(red for the $A^{+}$and blue for $X^{+}$state) and $\mathrm{H}_{2}{ }^{+}$ (green for both states) calculated with Eq. (11) for an approach in the $A^{+}$and $X^{+}$states are depicted in Fig. 8. As expected and already noticed in previous studies $[8,57,58]$, we find that the radiative association cross section $\sigma_{A^{+}}$is much larger than $\sigma_{X^{+}}$, because of the very limited number of bound rovibrational levels of the $A^{+}$state. The maximum values of the cross section (about $6 \times 10^{-6} a_{0}^{2}$ for both $\mathrm{H}_{2}{ }^{+}$

TABLE III. Probabilities $p_{i}$ for approaching in a given partial wave $N^{\prime}$ in the vibrational continuum of state $i=\left\{\mathrm{X}^{+}, A^{+}\right\}$for $\mathrm{H}_{2}{ }^{+}$and $\mathrm{D}_{2}{ }^{+}$.

\begin{tabular}{lccc}
\hline \hline$N^{\prime}$ & State & $\mathrm{H}_{2}{ }^{+}$ & $\mathrm{D}_{2}{ }^{+}$ \\
\hline \multirow{2}{*}{ Even } & $X^{+}$ & $1 / 4$ & $2 / 3$ \\
& $A^{+}$ & $3 / 4$ & $1 / 3$ \\
Odd & $X^{+}$ & $3 / 4$ & $1 / 3$ \\
& $A^{+}$ & $1 / 4$ & $2 / 3$ \\
\hline \hline
\end{tabular}




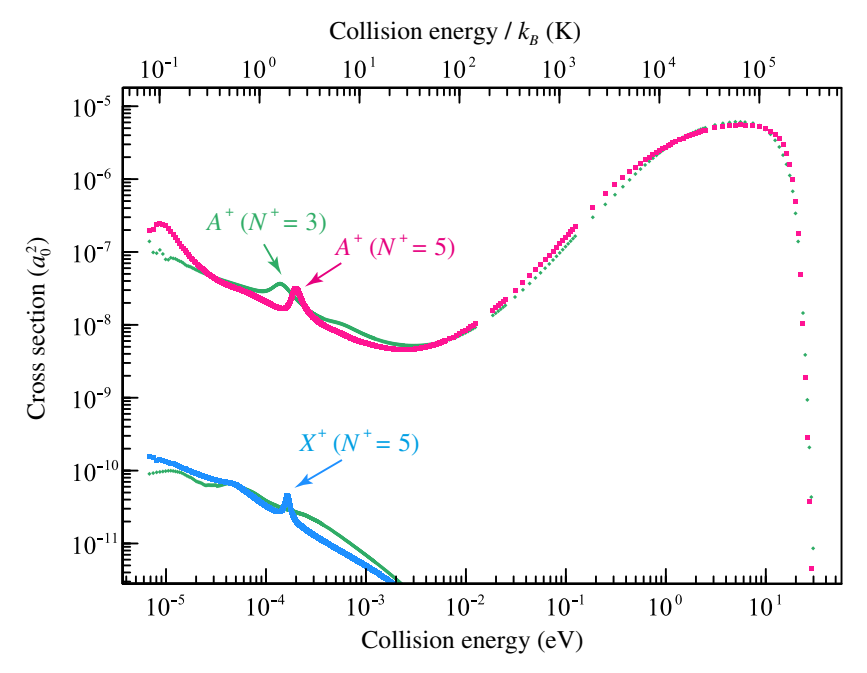

FIG. 8. Radiative-association cross sections as a function of the collision energy for $\mathrm{D}^{+}+\mathrm{D}(1 s)$ approaching in the $i=A^{+}$(red) or $i=X^{+}$(blue) entrance channel. The corresponding cross sections for $\mathrm{H}^{+}+\mathrm{H}(1 s)$ (green) are shown for comparison.

and $\mathrm{D}_{2}{ }^{+}$) are at $\sim 7 \mathrm{eV}$, where the classical inner turning point of the (repulsive) potential is located vertically above the equilibrium geometry of the $X^{+}$state.

At lower collision energies, Fig. 8 reveals an enhancement of $\sigma_{A^{+}}$resulting from the $A^{+}(0,5)$ shape resonance. A similar effect, though less prominent, is found in $\mathrm{H}_{2}{ }^{+}$and can be attributed to the $A^{+}(0,3)$ orbiting resonance. The cross sections for $\mathrm{H}_{2}{ }^{+}$agree with those published in Ref. [8], although deviations are found at lower collision energies. The differences in this region can be attributed to the use of Eq. (11) in Ref. [8] without taking the nuclearspin statistics into account. In $\mathrm{D}_{2}{ }^{+}$, an additional enhancement is observed at $0.1 \mathrm{~K}$ and can be attributed to the orbiting resonance $A^{+}(1,2)$ located at $\approx-0.065 \mathrm{~cm}^{-1}$ (see Fig. 7).

At low collision energies, the cross sections of the $X^{+} \rightarrow$ $A^{+}$process are almost 3 orders of magnitude smaller than for the $A^{+} \rightarrow X^{+}$process for both $\mathrm{D}_{2}{ }^{+}$and $\mathrm{H}_{2}{ }^{+}$. At higher collision energies, the cross sections further decrease because the slowly oscillating part of the $X^{+}$continuum wave functions shifts toward smaller internuclear distances. An enhancement of $\sigma_{X^{+}}\left(\mathrm{D}_{2}{ }^{+}\right)$at the position of the $X^{+}(26,5)$ resonance, which can radiate to the $A^{+}(0,4)$ bound state, is clearly visible. Although $\sigma_{X^{+}}\left(\mathrm{D}_{2}{ }^{+}\right)$is small in absolute numbers, the enhancement at $E_{\text {coll }}=$ $h c \cdot 1.336 \mathrm{~cm}^{-1}$ results from a single transition, i.e., $X^{+}(26,5) \rightarrow A^{+}(0,4)$ and might offer a possibility for the observation of $\mathrm{D}_{2}{ }^{+}$. The behavior of $\mathrm{D}_{2}{ }^{+}$differs from that of $\mathrm{H}_{2}{ }^{+}$, for which the lowest shape resonance $\left[X^{+}(18,4)\right.$, observed in Ref. [28]], cannot be stabilized by emitting a photon. Indeed, the $A^{+}$bound state with the largest rotational angular momentum is $A^{+}(0,2)$ and cannot be reached from $N^{\prime}=4$ in an electric-dipole transition.

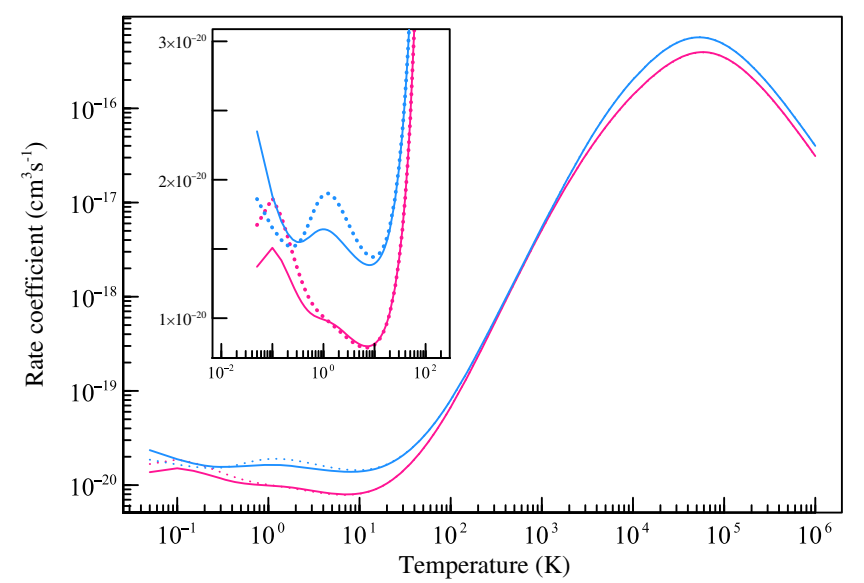

FIG. 9. Thermal rate coefficient for radiative association as a function of the temperature: $\mathrm{D}^{+}+\mathrm{D}(1 s)$ (red) and $\mathrm{H}^{+}+\mathrm{H}(1 s)$ (blue). The dotted lines correspond to calculations neglecting the restrictions imposed by the Pauli principle. The inset shows the curves at low temperatures on a linear scale.

The thermal rate coefficient $\alpha(T)$ at temperature $T$ can be calculated from the cross sections using [8]

$$
\begin{aligned}
\alpha(T)= & \left(\frac{8}{\pi \mu}\right)^{1 / 2}\left(\frac{1}{k_{B} T}\right)^{3 / 2} \\
& \times \int_{0}^{\infty} d E_{\text {coll }} \sigma\left(E_{\text {coll }}\right) E_{\text {coll }} \exp \left[-E_{\text {coll }} / k_{B} T\right],
\end{aligned}
$$

where $\sigma\left(E_{\text {coll }}\right)=\sigma_{A^{+}}\left(E_{\text {coll }}\right)+\sigma_{X^{+}}\left(E_{\text {coll }}\right)$ and is depicted in Fig. 9. The thermal averaging reduces the effects of the shape resonances, which are only noticeable in the rate coefficients as weak oscillations at temperatures below $1 \mathrm{~K}$. The rate coefficients for the formation of $\mathrm{H}_{2}{ }^{+}$are typically slightly larger than for $\mathrm{D}_{2}{ }^{+}$, an effect that is primarily caused by the larger reduced mass of $\mathrm{D}_{2}{ }^{+}$. However, a simple scaling with the reduced mass is not sufficient to obtain reliable rate constants for the formation of $\mathrm{D}_{2}{ }^{+}$from the rate coefficients calculated for $\mathrm{H}_{2}{ }^{+}$. For the formation of $\mathrm{H}_{2}{ }^{+}$, we find excellent agreement with the rate coefficients reported by Ramaker and Peek [7] above $20 \mathrm{~K}$. At lower temperatures, the rate coefficients can differ by as much as $15 \%$, primarily because the restrictions imposed by the Pauli principle were neglected in Ref. [7] (and also in Ref. [8]). The dotted lines in Fig. 9 correspond to calculations in which the restrictions imposed by the Pauli principle were neglected.

Given the very similar magnitudes of the rate coefficients for the formation of $\mathrm{H}_{2}{ }^{+}$and $\mathrm{D}_{2}{ }^{+}$, one may wonder why the recombination reaction $\mathrm{D}^{+}+\mathrm{D}$ is not considered in models of the chemistry in the early Universe [11,12]. Lepp et al. [12] for instance, consider 41 reactions involving $\mathrm{H}, \mathrm{D}, \mathrm{He}$, and $\mathrm{Li}$ in various charge states and present the relative abundances of molecular species in their Fig. 2 as a function of the red shift. The abundance of $\mathrm{H}_{2}{ }^{+}$is about 5 orders of magnitude larger than that of $\mathrm{HD}^{+}$at all values of 
the red shift. Given that $\mathrm{H}_{2}{ }^{+}$is thought to have been predominantly formed by radiative association in the early Universe [12] and that the ratio of $\mathrm{D}$ to $\mathrm{H}$ is about $10^{-5}$ [11], one might naively expect that the concentration of $\mathrm{D}_{2}{ }^{+}$ might be $10^{-10}$ that of $\mathrm{H}_{2}{ }^{+}$, which would still make it one of the most abundant molecules at high red shifts. Accurate values of the rate coefficients for the $\mathrm{H}^{+}+\mathrm{H}$ radiative association at low temperatures, such as those presented here, may also become relevant in future experiments aiming at forming the antimatter molecule $\overline{\mathrm{H}}_{2}{ }^{-}$made of a positron and two antiprotons.

\section{CONCLUSIONS}

We have presented a half-collision study of the $\mathrm{D}^{+}+\mathrm{D}$ and $\mathrm{H}^{+}+\mathrm{H}$ collision systems at low energies and high resolution by PFI-ZEKE photoelectron spectroscopy using a multiphoton excitation sequence through selected rotational levels of the $\bar{H}(v=12)$ intermediate level. In the PFI-ZEKE photoelectron spectra, we observed the lowlying shape resonances of $\mathrm{D}_{2}{ }^{+}$and $\mathrm{H}_{2}{ }^{+}$as sharp spectral structures superimposed onto a slowly varying signal resulting from the nodal structure of the wave function of the $\bar{H}$ state. The shape resonances were assigned on the basis of $a b$ initio calculations including nonadiabatic, relativistic, and radiative corrections as well as experiments in which the rotational quantum number of the intermediate state was changed from 0 to 6 .

Good agreement between experimental and calculated values of the positions and widths of all resonances was achieved after correction for the broadening of the resonances in the PFI-ZEKE photoelectron spectra resulting from $n$ changes caused by the localization of the charge of the dissociating ion core. This good agreement led to a validation of the calculated scattering phase shifts for both the $X^{+}$and $A^{+}$states, which are required to calculate scattering and radiative-association cross sections. In contrast to $\mathrm{H}_{2}{ }^{+}, \mathrm{D}_{2}{ }^{+}$possesses shape resonances in both the $X^{+}$and $A^{+}$state that have electric-dipole allowed transitions to bound states of $\mathrm{D}_{2}{ }^{+}$. The shape resonances of $\mathrm{D}_{2}{ }^{+}$thus play a role in the radiative-association reaction $\mathrm{D}^{+}+\mathrm{D} \rightarrow \mathrm{D}_{2}^{+}$at low temperature.

The cross sections and thermal rate coefficients presented in Figs. 7-9 cover a range of collision energies and temperatures spanning more than seven orders of magnitude. They correspond to a full quantum treatment, validated through comparison with experiment, and extend from the low-temperature regime, where the cross sections are dominated by quantum-mechanical effects, to the hightemperature regime, where their behavior is essentially classical.

\section{ACKNOWLEDGMENTS}

We thank Professor Jaquet for providing R-dependent vibrational and rotational reduced masses for the hydrogen molecular ion in numerical form. This work was supported financially by the Swiss National Science Foundation (Project No. 200020-172620) and the European Research Council (ERC) under the European Union's Horizon 2020 research and innovation programme (Advanced Grant No. 743121).

[1] M. T. Bell and T.P. Softley, Ultracold Molecules and Ultracold Chemistry, Mol. Phys. 107, 99 (2009).

[2] D. W. Chandler, Cold and Ultracold Molecules: Spotlight on Orbiting Resonances, J. Chem. Phys. 132, 110901 (2010).

[3] A. B. Henson, S. Gersten, Y. Shagam, J. Narevicius, and E. Narevicius, Observation of Resonances in Penning Ionization Reactions at sub-Kelvin Temperatures in Merged Beams, Science 338, 234 (2012).

[4] C. Naulin and M. Costes, Experimental Search for Scattering Resonances in Near Cold Molecular Collisions, Int. Rev. Phys. Chem. 33, 427 (2014).

[5] S. N. Vogels, J. Onvlee, S. Chefdeville, A. v. d. Avoird, G. C. Groenenboom, and S. Y. T. v. d. Meerakker, Imaging Resonances in Low-Energy NO-He Inelastic Collisions, Science 350, 787 (2015).

[6] J. Jankunas, K. Jachymski, M. Hapka, and A. Osterwalder, Communication: Importance of Rotationally Inelastic Processes in Low-Energy Penning Ionization of $\mathrm{CHF}_{3}$, J. Chem. Phys. 144, 221102 (2016).

[7] D. E. Ramaker and J. M. Peek, Molecule Formation in Tenuous Media: Quantum Effects in Spontaneous Radiative aAssociation, Phys. Rev. A 13, 58 (1976).

[8] P. C. Stancil, J. F. Babb, and A. Dalgarno, The Radiative Association of $\mathrm{He}^{+}$and $\mathrm{He}$ and $\mathrm{H}^{+}$and $\mathrm{H}$, Astrophys. J. 414, 672 (1993).

[9] S. Lepp and J. M. Shull, Molecules in the Early Universe, Astrophys. J. 280, 465 (1984).

[10] P. C. Stancil, S. Lepp, and A. Dalgarno, The Deuterium Chemistry of the Early Universe, Astrophys. J. 509, 1 (1998).

[11] D. Galli and F. Palla, The Chemistry of the Early Universe, Astron. Astrophys. 335, 403 (1998).

[12] S. Lepp, P. C. Stancil, and A. Dalgarno, Atomic and Molecular Processes in the Early Universe, J. Phys. B 35, R57 (2002).

[13] C. M. Coppola, D. Galli, F. Palla, S. Longo, and J. Chluba, Non-Thermal Photons and $\mathrm{H}_{2}$ Formation in the Early Universe, Mon. Not. R. Astron. Soc. 434, 114 (2013).

[14] A. T. Grier, M. Cetina, F. Oručević, and V. Vuletić, Observation of Cold Collisions between Trapped Ions and Trapped Atoms, Phys. Rev. Lett. 102, 223201 (2009).

[15] C. Zipkes, S. Palzer, C. Sias, and M. Köhl, A Trapped Single Ion inside a Bose-Einstein Condensate, Nature (London) 464, 388 (2010).

[16] S. Schmid, A. Härter, and J. H. Denschlag, Dynamics of a Cold Trapped Ion in a Bose-Einstein Condensate, Phys. Rev. Lett. 105, 133202 (2010).

[17] F. H. J. Hall, M. Aymar, N. Bouloufa-Maafa, O. Dulieu, and S. Willitsch, Light-Assisted Ion-Neutral Reactive Processes 
in the Cold Regime: Radiative Molecule Formation versus Charge Exchange, Phys. Rev. Lett. 107, 243202 (2011).

[18] T. Sikorsky, M. Morita, Z. Meir, A. A. Buchachenko, R. Ben-shlomi, N. Akerman, E. Narevicius, T. V. Tscherbul, and R. Ozeri, Phase-Locking between Different PartialWaves in Atom-Ion Spin-Exchange Collisions, arXiv: 1806.05150 .

[19] D. Gerlich, Experimental Investigations of Ion-Molecule Reactions Relevant to Interstellar Chemistry, J. Chem. Soc., Faraday Trans. 89, 2199 (1993).

[20] I. W. M. Smith and B. R. Rowe, Reaction Kinetics at Very Low Temperatures: Laboratory Studies and Interstellar Chemistry, Acc. Chem. Res. 33, 261 (2000).

[21] A. Carrington, I. R. McNab, and C. A. Montgomerie, Spectroscopy of the Hydrogen Molecular Ion, J. Phys. B 22, 3551 (1989).

[22] A. Carrington, I. R. McNab, C. A. Montgomerie, and R. A. Kennedy, Electronic Spectrum $2 p \sigma_{u}-1 s \sigma_{g}$ of the $\mathrm{D}_{2}{ }^{+}$Ion, Mol. Phys. 67, 711 (1989).

[23] A. Carrington, I. R. McNab, C. A. Montgomerie, and J. M. Brown, Microwave Spectra of the $\mathrm{HD}^{+}$and $\mathrm{D}_{2}^{+}$Ions at their Dissociation Limits, Mol. Phys. 66, 1279 (1989).

[24] A. Carrington, C. A. Leach, A. J. Marr, R. E. Moss, C. H. Pyne, and T. C. Steimle, Microwave Spectra of the $\mathrm{D}_{2}{ }^{+}$and $\mathrm{HD}^{+}$Ions near their Dissociation Limits, J. Chem. Phys. 98, 5290 (1993).

[25] A. Carrington, D. I. Gammie, A. M. Shaw, and S. M. Taylor, Millimetre-Wave Electronic Spectrum of the $\mathrm{D}_{2}{ }^{+}$Ion, J. Chem. Soc., Faraday Trans. 91, 1887 (1995).

[26] R. E. Moss, Calculations for the Vibration-Rotation Levels of $\mathrm{H}_{2}{ }^{+}$in its Ground and First Excited Electronic States, Mol. Phys. 80, 1541 (1993).

[27] P. S. Krstić, J. H. Macek, S. Y. Ovchinnikov, and D. R. Schultz, Analysis of Structures in the Cross Sections for Elastic Scattering and Spin Exchange in Low-Energy $\mathrm{H}^{+}+\mathrm{H}$ Collisions, Phys. Rev. A 70, 042711 (2004).

[28] M. Beyer and F. Merkt, Observation and Calculation of the Quasibound Rovibrational Levels of the Electronic Ground State of $\mathrm{H}_{2}{ }^{+}$, Phys. Rev. Lett. 116, 093001 (2016).

[29] M. Beyer and F. Merkt, Structure and Dynamics of $\mathrm{H}_{2}{ }^{+}$ near the Dissociation Threshold: A Combined Experimental and Computational Investigation, J. Mol. Spectrosc. 330, 147 (2016).

[30] K. Müller-Dethlefs and E. W. Schlag, Chemical Applications of Zero Kinetic Energy (ZEKE) Photoelectron Spectroscopy, Angew. Chem. 37, 1346 (1998).

[31] F. Merkt, S. Willitsch, and U. Hollenstein, in Handbook of High-Resolution Spectroscopy, edited by M. Quack and F. Merkt (John Wiley \& Sons, Chichester, 2011), Vol. 3, pp. 1617-1654.

[32] V. I. Korobov, Ro-Vibrational States of $\mathrm{H}_{2}{ }^{+}$. Variational Calculations, Mol. Phys. 116, 93 (2018).

[33] V. A. Belyaev, B. G. Brezhnev, and E. M. Erastov, Resonance Charge Exchange of Protons and Deuterons at Low Energies, Sov. Phys. JETP 25, 777 (1967).

[34] R. E. Moss, The $2 p \sigma_{u}-1 s \sigma_{g}$ Electronic Spectrum of $\mathrm{D}_{2}{ }^{+}$, J. Chem. Soc., Faraday Trans. 89, 3851 (1993).

[35] L. Wolniewicz and T. Orlikowski, The $1 s \sigma_{g}$ and $2 p \sigma_{u}$ States of the $\mathrm{H}_{2}^{+}, \mathrm{D}_{2}^{+}$and $\mathrm{HD}^{+}$Ions, Mol. Phys. 74, 103 (1991).
[36] M. Beyer and F. Merkt, High-Resolution Photoelectron Spectroscopy and Calculations of the Highest Bound Levels of $\mathrm{D}_{2}{ }^{+}$below the First Dissociation Threshold, J. Phys. B 50, 154005 (2017).

[37] J. Liu, D. Sprecher, Ch. Jungen, W. Ubachs, and F. Merkt, Determination of the Ionization and Dissociation Energies of the Deuterium Molecule $\left(\mathrm{D}_{2}\right)$, J. Chem. Phys. 132, 154301 (2010).

[38] U. Hollenstein, R. Seiler, H. Schmutz, M. Andrist, and F. Merkt, Selective Field Ionization of High Rydberg States: Application to Zero-Kinetic-Energy Photoelectron Spectroscopy, J. Chem. Phys. 115, 5461 (2001).

[39] R. Jaquet and M. V. Khoma, Nonadiabatic Investigations of Ro-Vibrational Frequencies within the Systems $\mathrm{H}_{2}{ }^{+}, \mathrm{H}_{2}$, and Prospects for $\mathrm{H}_{3}{ }^{+}$: Use of Distance-Dependent Effective Masses, Mol. Phys. 110, 669 (2012).

[40] R. Jaquet and W. Kutzelnigg, Non-Adiabatic Theory in Terms of a Single Potential Energy Surface. The VibrationRotation Levels of $\mathrm{H}_{2}{ }^{+}$and $\mathrm{D}_{2}{ }^{+}$, Chem. Phys. 346, 69 (2008).

[41] F. T. Smith, Lifetime Matrix in Collision Theory, Phys. Rev. 118, 349 (1960).

[42] J. P. Davis and W. R. Thorson, Very Low Energy Scattering in $\mathrm{HH}^{+}$and $\mathrm{HD}^{+}$, Can. J. Phys. 56, 996 (1978).

[43] L. Wolniewicz, The $H \bar{H}{ }^{1} \Sigma_{g}^{+}$State of the Hydrogen Molecule, J. Chem. Phys. 108, 1499 (1998).

[44] A. Lindgard and S. E. Nielsen, Numerical Approach to Transit Probabilities in the Coulomb Approximation: Be II and Mg II Rydberg Series, J. Phys. B 8, 1183 (1975).

[45] A. Matzkin, C. Jungen, and S. C. Ross, Extended Coulomb Approximation for Multichannel-Quantum-Defect-Theory Computations of Dipole Moments: Method of Calculation and Application to $\mathrm{H}_{2}$, Phys. Rev. A 58, 4462 (1998).

[46] D. R. Bates and D. A. Williams, Low Energy Collisions between Hydrogen Atoms and Protons, Proc. Phys. Soc. London 83, 425 (1964).

[47] G. Hunter and M. Kuriyan, Scattering of Protons by Hydrogen Atoms at Low Energies: Phase Shifts and Differential Cross Sections, At. Data Nucl. Data Tables 25, 287 (1980).

[48] F. Masnou-Seeuws and A. Salin, Collision Problems with Identical Particles, J. Phys. B 2, 1274 (1969).

[49] P. S. Krstić and D. R. Schultz, Consistent Definitions for, and Relationships among, Cross Sections for Elastic Scattering of Hydrogen Ions, Atoms, and Molecules, Phys. Rev. A 60, 2118 (1999).

[50] W. A. Chupka, Factors Affecting Lifetimes and Resolution of Rydberg States Observed in Zero-Electron-KineticEnergy Spectroscopy, J. Chem. Phys. 98, 4520 (1993).

[51] F. Merkt and R. N. Zare, On the Lifetimes of Rydberg States Probed by Delayed Pulsed Field Ionization, J. Chem. Phys. 101, 3495 (1994).

[52] T.F. Gallagher, Rydberg Atoms (Cambridge University Press, Cambridge, 1994).

[53] E. Wells, K. D. Carnes, B. D. Esry, and I. Ben-Itzhak, Charge Transfer and Elastic Scattering in Very Slow $\mathrm{H}^{+}+$ D(1s) Half Collisions, Phys. Rev. Lett. 86, 4803 (2001).

[54] J. Xie and R. N. Zare, Selection Rules for the Photoionization of Diatomic Molecules, J. Chem. Phys. 93, 3033 (1990). 
[55] U. Fano and A. Rau, Atomic Collisions and Spectra (Academic Press, Orlando, 1986).

[56] D. R. Bates, Rate of Formation of Molecules by Radiative Association, Mon. Not. R. Astron. Soc. 111, 303 (1951).

[57] J.F. Babb and A. Dalgarno, Radiative Association and Inverse Predissociation of Oxygen Atoms, Phys. Rev. A 51, 3021 (1995).
[58] J. F. Babb and K. P. Kirby, in Molecular Astrophysics of Stars and Galaxies, edited by T. W. Hartquist and D. A. Williams (Clarendon, Oxford, 1998), p. 11.

[59] R. S. Mulliken, Intensities of Electronic Transitions in Molecular Spectra II. Charge Transfer Spectra, J. Chem. Phys. 7, 20 (1939).

[60] D. R. Bates, The Oscillator Strength of $\mathrm{H}_{2}{ }^{+}, 1 s \sigma-2 p \sigma$, J. Chem. Phys. 19, 1122 (1951). 